\title{
Interference Alignment and the Degrees of Freedom of Wireless $X$ Networks
}

\author{
Viveck R. Cadambe, Syed A. Jafar \\ Center for Pervasive Communications and Computing \\ Electrical Engineering and Computer Science \\ University of California Irvine, \\ Irvine, California, 92697, USA \\ Email:vcadambe@uci.edu, syed@uci.edu
}

\begin{abstract}
We explore the degrees of freedom of $M \times N$ user wireless $X$ networks, i.e. networks of $M$ transmitters and $N$ receivers where every transmitter has an independent message for every receiver. We derive a general outerbound on the degrees of freedom region of these networks. When all nodes have a single antenna and all channel coefficients vary in time or frequency, we show that the total number of degrees of freedom of the $X$ network is equal to $\frac{M N}{M+N-1}$ per orthogonal time and frequency dimension. Achievability is proved by constructing interference alignment schemes for $X$ networks that can come arbitrarily close to the outerbound on degrees of freedom. For the case where either $M=2$ or $N=2$ we find that the degrees of freedom characterization also provides a capacity approximation that is accurate to within $O(1)$. For these cases the degrees of freedom outerbound is exactly achievable.
\end{abstract}




\section{INTRODUCTION}

There is increasing interest in approximate capacity characterizations of wireless networks as a means to understanding their performance limits. In particular, the high SNR regime - where the local additive white Gaussian noise (AWGN) at each node is de-emphasized relative to signal and interference powers - offers fundamental insights into optimal interference management schemes. The degrees-of-freedom approach provides a capacity approximation whose accuracy approaches $100 \%$ in the high signal-to-noise ratio (SNR) regime. A network has $d$ degrees of freedom if and only if the sum capacity of the network can be expressed as $d \log (S N R))+o(\log (S N R))$. Since each orthogonal (non-interfering) signalling dimension contributes a rate of $\log (S N R)+o(\log (S N R))$, the degrees of freedom of a network may be interpreted as the number of resolvable signal space dimensions. The capacity characterizations obtained through this approach are equivalently described by various researchers as the multiplexing gain, the pre-log term or the degrees of freedom characterization. Starting with the point to point MIMO channel [1], [2], the degrees of freedom have been characterized for MIMO multiple access channel (MAC) [3], MIMO broadcast channel (BC) [4]-[6], 2 user MIMO interference channel [7], various distributed relay networks [8]-[10], 2 user MIMO $X$ channel [11]-[15], and most recently the $K$ user interference channel [16]. For the purpose of this paper, relevant ideas from these prior works are summarized as follows.

Consider a two user Gaussian interference channel where each node is equipped with a single antenna. Transmitters $T_{1}$ and $T_{2}$ have independent messages $W_{11}$ and $W_{22}$ for receivers $R_{1}$ and $R_{2}$, respectively. It is known that if transmitters $T_{1}$ and $T_{2}$ are combined into one compound transmitter with 2 transmit antennas and (or) the receivers $R_{1}$ and $R_{2}$ are combined into one compound receiver with 2 receive antennas, then the resulting point to point MIMO channel (or the resulting vector MAC/BC) has 2 degrees of freedom, i.e. the sum capacity of the resulting channel is expressed as $2 \log (S N R)+o(\log (S N R))$ [3]-[6]. However, the interference channel with distributed transmitters and distributed receivers has only 1 degree of freedom, i.e., its sum capacity is only $\log (S N R)+o(\log (S N R))$ [7], [17]. This loss of degrees of freedom is evidently due to the inability of the transmitters/receivers to jointly process the transmitted/received signals.

Consider again the same two user network with distributed transmitters and receivers, but suppose there are four independent messages $W_{11}, W_{12}, W_{21}, W_{22}$ such that message $W_{i j}$ originates at transmitter $j$ and is intended for receiver $i$. This communication scenario is named the $X$ channel in [18] and is shown to have $\frac{4}{3}$ degrees of freedom in [15] when the channel coefficients are time-varying or frequency-selective and drawn from a continuous distribution. The degrees of freedom of the constant $X$ channel (i.e. when the channel coefficients are not timevarying or frequency-selective) remain unknown in general for single antenna nodes ${ }^{1}$. A key concept that arises in the context of the $X$ channel [11], [15] (also an essential element of wireless interference networks [16], [19]-[22] and the compound broadcast channel [23]), is the idea of "Interference Alignment" that refers to an overlap of signal spaces occupied by undesired interference at each receiver while keeping the desired signal spaces distinct. To illustrate why interference alignment on the $\mathrm{X}$ channel leads to $4 / 3$ degrees of freedom, we construct the following simple example where alignment is achieved in terms of signal propagation delays.

\footnotetext{
${ }^{1}$ The constant $\mathrm{X}$ channel with $M>1$ antennas at each node is considered in [11] and shown to achieve $\left\lfloor\frac{4 M}{3}\right\rfloor$ degrees of freedom. The result is strengthened in [15] where achievability of $\frac{4 M}{3}$ degrees of freedom is established along with a converse.
} 


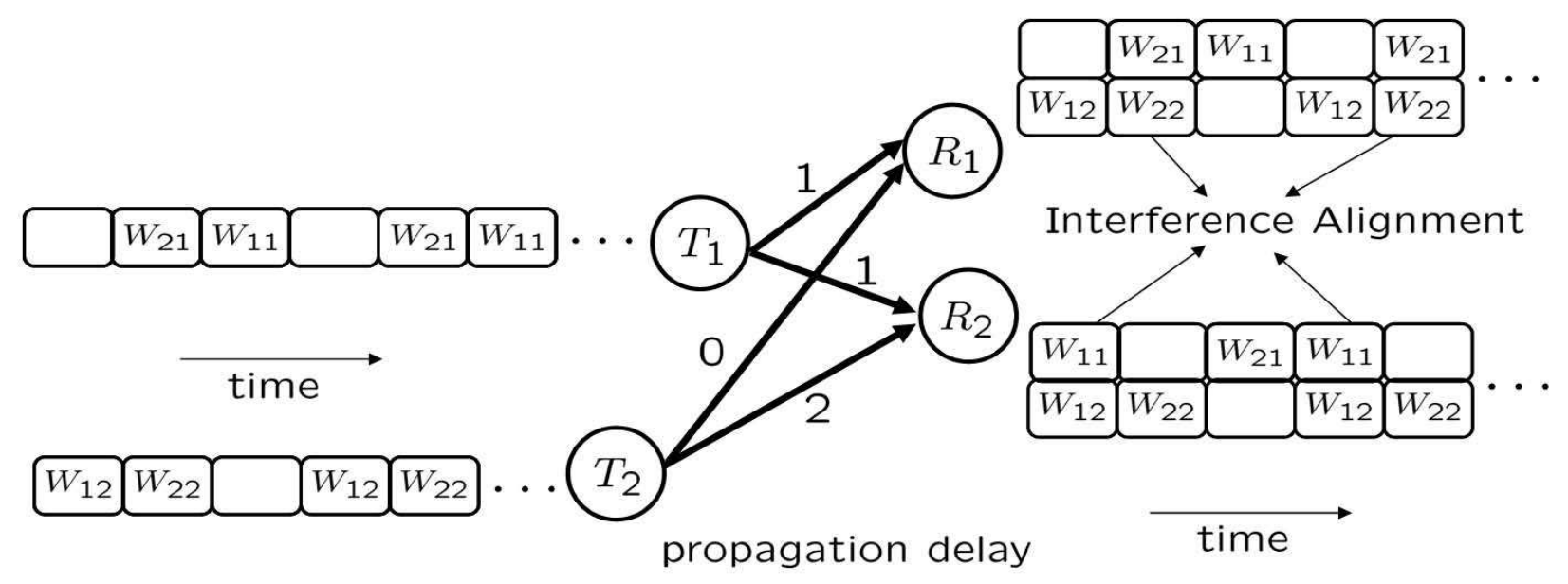

Fig. 1. Interference Alignment on the $X$ channel. 4 interference-free channels are created over 3 time slots

\section{Toy Example - 4 orthogonal channels over 3 time slots}

As shown in Figure 1, in this toy example we assume there is a propagation delay between each transmitter and receiver (the delay 0 can also be interpreted as a delay of 3 time slots). The transmissions are scheduled such that, following the propagation delays, they arrive at the desired receivers free from interference, while they are aligned with other interference at the undesired receivers. Thus, for example, transmitted symbols for message $W_{11}$ are received free from interference at intended receiver $R_{1}$ but interfere with the symbols for message $W_{12}$ at receiver 2 since both $W_{11}, W_{12}$ are undesired messages for $R_{2}$. Due to this interference alignment, all 4 messages are delivered interference-free to their respective destinations using only 3 time slots. While the toy example uses artificial propagation delays, essentially the same alignment is accomplished in [15] (without involving propagationdelays) over random time-varying channels by sophisticated beamforming in space and time dimensions. Thus, the toy example illustrates the basic idea behind the $4 / 3$ degrees of freedom of the 2 user $X$ channel $^{2}$.

Interference alignment is the key to the degrees of freedom characterizations of a variety of network communication scenarios, such as the compound broadcast channel [23], cognitive radio networks [15], [26], deterministic channel models [24] and the interference channel with $K>2$ users [16]. While the principle of interference alignment is quite simple, the extent to which interference can be aligned for a general network topology is difficult to determine. Ideally one would like all interfering signals to align at every receiver and all desired signals to be distinguishable. As we introduce more messages into the network, the interference alignment problem becomes increasingly complex. The most challenging case for interference alignment is therefore the $X$ network where every transmitter has an independent message for every receiver. In this paper we explore this extreme scenario to find out the limits of interference alignment.

\footnotetext{
${ }^{2}$ As pointed out in [24], propagation delay based examples such as Figure 1 can be translated first into the deterministic channel model of [25] and then into a constant $X$ channel with certain specific channel coefficients which can achieve arbitrarily close to the outer bound of $4 / 3$ degrees of freedom.
} 


\section{Definitions - $X$ Network And Degrees of Freedom}

Following the terminology of [18], we define an $M \times N$ user $X$ network as a communication network with $M$ transmitters and $N$ receivers and a total of $M N$ independent messages, one from each transmitter to each receiver. The transmitters cannot receive and receivers cannot transmit which precludes relaying, feedback and cooperation between transmitters or cooperation between receivers. A $2 \times 3$ user $X$ network is shown in Figure 2 . The $M \times N$ user $X$ network is described by input-output relations

$$
Y^{[j]}(\kappa)=\sum_{i \in\{1,2, \ldots, M\}} H^{[j i]}(\kappa) X^{[i]}(\kappa)+Z^{[j]}(\kappa), \quad j=1,2, \ldots, N
$$

where $\kappa$ represents the channel use index. For simplicity we will assume $\kappa$ represents the time index. It should be noted that it can equivalently be interpreted as the frequency index if coding occurs over orthogonal frequency slots. $X^{[i]}(\kappa)$ is the signal transmitted by transmitter $i, Y^{[j]}(\kappa)$ is the signal received by receiver $j$ and $Z^{[j]}(\kappa)$ represents the additive white Gaussian noise at receiver $j$. The noise variance at all receivers is assumed to be equal to unity. $H^{[j i]}(\kappa)$ represents the channel gain between transmitter $i$ and receiver $j$ at time $\kappa$. We assume that all channel fade coefficients are drawn according to a continuous distribution. Specifically, we assume that the cumulative distribution function

$$
F_{i, j, \kappa}(h)=\operatorname{Pr}\left(H^{[j i]}(\kappa) \leq h \mid\left\{H^{\left[j^{\prime} i^{\prime}\right]}\left(\kappa^{\prime}\right),\left(i^{\prime}, j^{\prime}, \kappa^{\prime}\right) \neq(i, j, \kappa)\right\}\right)
$$

is continuous in $h$. Further, to avoid degenerate channel conditions, we assume that the absolute value of all the channel gains is bounded between a non-zero minimum value and a finite maximum value. We assume that all nodes have causal (i.e, present and past) knowledge of all the channel gains, meaning that at time index $\kappa$, each node knows all the elements of the set $\left\{H^{[j i]}(k):(j, i) \in\{1,2, \ldots, N\} \times\{1,2, \ldots, M\}, k=1,2, \ldots, \kappa\right\}$.

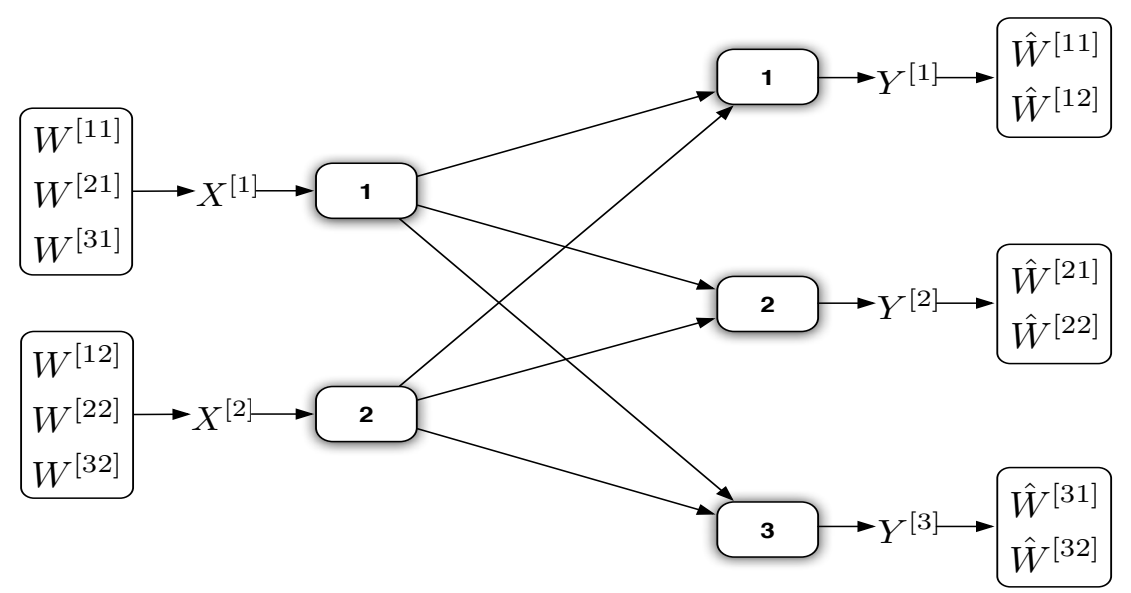

Fig. 2. A $2 \times 3$ user $X$ network

We assume that transmitter $i$ has message $W^{[j i]}$ for receiver $j$, for each $i \in\{1,2, \ldots, M\}, j \in\{1,2, \ldots, N\}$, resulting in a total of $M N$ mutually independent messages. The average power at each transmitter is bounded by $\rho$, i.e.

$$
E\left(\frac{1}{\kappa_{0}} \sum_{\kappa=1}^{\kappa_{0}}\left|X^{[i]}(\kappa)\right|^{2}\right) \leq \rho
$$


for all $i \in\{1,2, \ldots, M\}$, where $\kappa_{0}$ denotes the length of the codeword. Let $R_{j i}(\rho)=\frac{\log \left(\left|W^{[j i]}(\rho)\right|\right)}{\kappa_{0}}$ denote the rate of the codeword encoding the message $W^{[j i]}$, where $\left|W^{[j i]}(\rho)\right|$ denotes the size of the message set. A ratematrix $\left[\left(R_{j i}(\rho)\right)\right]$ is said to be achievable if messages $W^{[j i]}$ can be encoded at rates $R_{j i}(\rho)$ so that the probability of error can be made arbitrarily small simultaneously for all messages by choosing appropriately long $\kappa_{0}$. Let $\mathcal{C}(\rho)$ represent capacity region of the $X$ network, i.e, it represents the set of all achievable rate-matrices $\left[\left(R_{j i}(\rho)\right)\right]$. Analogous to the capacity region of the network, the degrees of freedom region of the $M \times N$ user $X$ network is defined by

$$
\begin{aligned}
\mathcal{D}=\left\{\left[\left(d_{j i}\right)\right] \in \mathbb{R}_{+}^{M N}: \forall\left[\left(w_{j i}\right)\right] \in \mathbb{R}_{+}^{M N}\right. & \left.\sum_{i \in\{1,2, \ldots, M\}, j \in\{1,2, \ldots, N\}} w_{j i} d_{j i} \leq \lim \sup _{\rho \rightarrow \infty}\left[\sup _{\left[\left(R_{j i}(\rho)\right)\right] \in \mathcal{C}(\rho)} \sum_{i \in\{1,2, \ldots, M\}, j \in\{1,2, \ldots, N\}}\left[w_{j i} R_{j i}(\rho)\right] \frac{1}{\log (\rho)}\right]\right\}
\end{aligned}
$$

The degrees of freedom region of the network approximates its capacity region within $o(\log (\rho)) . X$ networks are interesting because they encompass all communication scenarios possible in a one-way single hop wireless network. For example, multiple access, broadcast and interference networks are special cases of $X$ networks. Since there are messages from every transmitter to every receiver, every transmitter is associated with a broadcast channel, every receiver is associated with a multiple access channel and every disjoint pairing of transmitters and receivers comprises an interference channel within the $X$ network. In particular, any outerbound on the degrees of freedom region of an $X$ network is also an outerbound on the degrees of freedom of all its subnetworks.

\section{RESULTS}

A summary of the key results and the associated insights is presented in this section.

1) Outerbound: The first result of this paper, presented in Section IV, is an outerbound for the degrees of freedom region of the $M \times N$ user $X$ network. In particular, the total number of degrees of freedom of the $M \times N$ user $X$ network is shown to be upper-bounded by $\frac{A M N}{M+N-1}$ per orthogonal time and frequency dimension, when each node is equipped with $A$ antennas. The outerbound is quite general as it applies to any fully connected (i.e. all channel coefficients are non-zero) $M \times N$ user $X$ network, regardless of whether the channel coefficients are constant or time varying. The key to the outerbound is to distribute the $M N$ messages in the $X$ network into $M N$ (partially overlapping) sets, each having $M+N-1$ elements. By picking these sets in a certain manner we are able to derive a MAC (multiple access channel) outerbound similar to [7] for the sum rate of the messages in each set. Since the MAC receiver has only $A$ antennas, the MAC has at most $A$ degrees of freedom. Thus, each set of messages can at most have $A$ degrees of freedom. The outerbounds for these sets together define an outerbound on the degrees of freedom region of the $M \times N$ user $X$ network and adding all the outerbounds gives us the bound on the total number of degrees of freedom.

2) Asymptotic Interference Alignment Scheme: In Section V-C we present an asymptotic interference alignment scheme for $M \times N$ user $X$ networks with time varying channel coefficients. By considering larger supersymbols the partial interference alignment scheme is able to approach within any $\epsilon>0$ of the degrees of freedom outerbound. While the idea of partially aligning interference was earlier used in the $K$ user interference channel [16], the extension of the scheme to the $X$ network is more complex, since there is a message for each transmitter-receiver pair in the network. Combined with the outerbound, the partial interference alignment scheme establishes that 
the total number of degrees of freedom of $M \times N$ user $X$ networks with single antenna nodes and time (or frequency) varying channel coefficients is precisely $\frac{M N}{M+N-1}$. The partial interference alignment scheme does not extend completely to $X$ networks where each node has multiple antennas. However, if we imagine each antenna to be a separate user (which can only reduce the capacity) then a simple application of the partial interference alignment scheme shows that an innerbound of $\frac{A M N}{M+N-1 / A}$ is achievable for $M \times N$ user $X$ networks where each node has $A$ antennas. If either $M$ or $N$ is reasonably large, then this innerbound is close to the outerbound.

3) Perfect Interference Alignment Scheme: We construct a perfect interference alignment scheme for the $M \times N$ user $X$ channel when the number of receivers $N=2$. This scheme achieves exactly one degree of freedom for every message over an $M+N-1$ symbol extension of the channel, thus achieving exactly the outerbound of $\frac{M N}{M+N-1}$ total degrees of freedom over a finite channel extension. We also show an interesting reciprocity property of beamforming and zero-forcing based schemes in wireless networks. In particular, we show that given a coding scheme in the $X$ network based entirely on beamforming and zero-forcing, we can construct a beamforming and zero-forcing based coding scheme over the reciprocal $X$ network achieving the same number of degrees of freedom as the original scheme. The coding scheme over the reciprocal channel may need apriori knowledge of all channel gains even when the original scheme needs only causal channel knowledge. The reciprocal scheme is therefore practical in a scenario where channel extensions are considered in the frequency domain. This reciprocity property serves as an achievability proof for the $M \times N$ user $X$ channel when $M=2$, for any $N$. Thus, for either $M=2$ or $N=2$ we are able to construct perfect interference alignment schemes with a finite extension of the channel. We show that this implies that, in both these cases, we have an $O(1)$ capacity characterization. Note that the asymptotic interference alignment scheme for the general case only yields a capacity characterization within $o(\log (S N R))$.

4) $X$ networks versus Interference Networks: Since we are able to characterize the exact degrees of freedom of $X$ networks and the degrees of freedom of interference networks are already known, the comparison follows simply as a corollary. The $K \times K$ user $X$ channel has significant degrees of freedom advantage over the $K$ user interference channel when $K$ is small. For example, when $K=2$, the $X$ network has $4 / 3$ degrees of freedom, whereas the interference channel has only 1 . However the advantage disappears as $K$ increases. This is easily seen by substituting $M=N=K$ in the total degrees of freedom expression for the $X$ channel to obtain $\frac{K^{2}}{2 K-1}$ which is close to $K / 2$ for large $K$.

5) Cost of Distributed Processing: This result also follows as a corollary of the main result that establishes the degrees of freedom for $X$ networks. Compared to $M \times N$ MIMO which represents joint signal processing at all transmitters and all receivers, the $M \times N$ user $X$ channel pays a degrees of freedom penalty of $\min (M, N)-\frac{M N}{M+N-1}$, which is the cost of distributed processing on the $X$ channel. While the cost of distributed processing is equal to half the degrees of freedom on the interference channel, it is interesting to note that for $X$ networks, this penalty disappears when the number of transmitters is much larger than the number of receivers or vice versa. This is easily seen because, when $M \gg N$ or $N \gg M, \frac{M N}{M+N-1}$ is very close to $\min (M, N)$. In other words, a small set of distributed nodes in a wireless communication network with no shared messages can serve as a multi-antenna node, if they are transmitting to, or receiving from a large number of distributed nodes. We also provide an application of this result - the two-hop parallel relay network with $M$ distributed transmitting and receiving nodes with large number of relays. In [8], this parallel relay network is shown to have $M / 2$ degrees of freedom if the number of relays was large. By treating the network as a compound of a $M \times K$ and a $K \times M X$ channel, we construct an alternate degrees-of-freedom-optimal achievable scheme in section VI. 


\section{Degrees of Freedom Region Outerbound for $X$ Networks}

While our main focus in this paper is on the case where each node has a single antenna, we present the outerbound for the more general setting where transmitter $i$ has $A_{i}^{t}$ antennas and receiver $j$ has $A_{j}^{r}$ antennas, $\forall i \in\{1,2, \cdots, M\}, j \in\{1,2, \cdots, N\}$.

Theorem 1: Let

$$
\begin{aligned}
& \mathcal{D}^{\text {out }} \triangleq\left\{\left[\left(d_{j i}\right)\right]: \forall(m, n) \in\{1,2, \ldots, M\} \times\{1,2, \ldots, N\}\right. \\
&\left.\sum_{q=1}^{N} d_{q m}+\sum_{p=1}^{M} d_{n p}-d_{n m} \leq \max \left(A_{m}^{t}, A_{n}^{r}\right)\right\}
\end{aligned}
$$

Then $\mathcal{D} \subseteq \mathcal{D}^{\text {out }}$ where $\mathcal{D}$ represents the degrees of freedom region of the $M \times N$ user $X$ channel. In other words, for any achievable scheme, the number of degrees of freedom achieved by all the messages associated with transmitter $m$ or receiver $n$ is upper-bounded by $\max \left(A_{m}^{t}, A_{n}^{r}\right)$.

Proof: We start by defining $M N$ sets $\mathcal{W}^{n m}, n \in\{1,2, \cdots, N\}, m \in\{1,2, \cdots, M\}$ as follows:

$$
\mathcal{W}^{n m} \triangleq\left\{W^{[p q]}:(p-n)(q-m)=0\right\}
$$

In other words, the set $\mathcal{W}^{n m}$ contains only those messages that either originate at transmitter $m$ or are destined for receiver $n$. Note that the $M N$ sets are not disjoint and that each set contains $M+N-1$ elements.

We will determine an outerbound for the total degrees of freedom achievable by each of the message sets when all other messages are eliminated. In other words, consider the $X$ channel when the only messages that need to be communicated are those that belong to the set $\mathcal{W}^{n m}$. Note that eliminating some messages cannot hurt the rates achievable by the remaining messages, as shown in [15], [16]. Now we show that the total number of degrees of freedom of all messages in a set $\mathcal{W}^{n m}$ is no more than $\max \left(A_{m}^{t}, A_{n}^{r}\right)$.

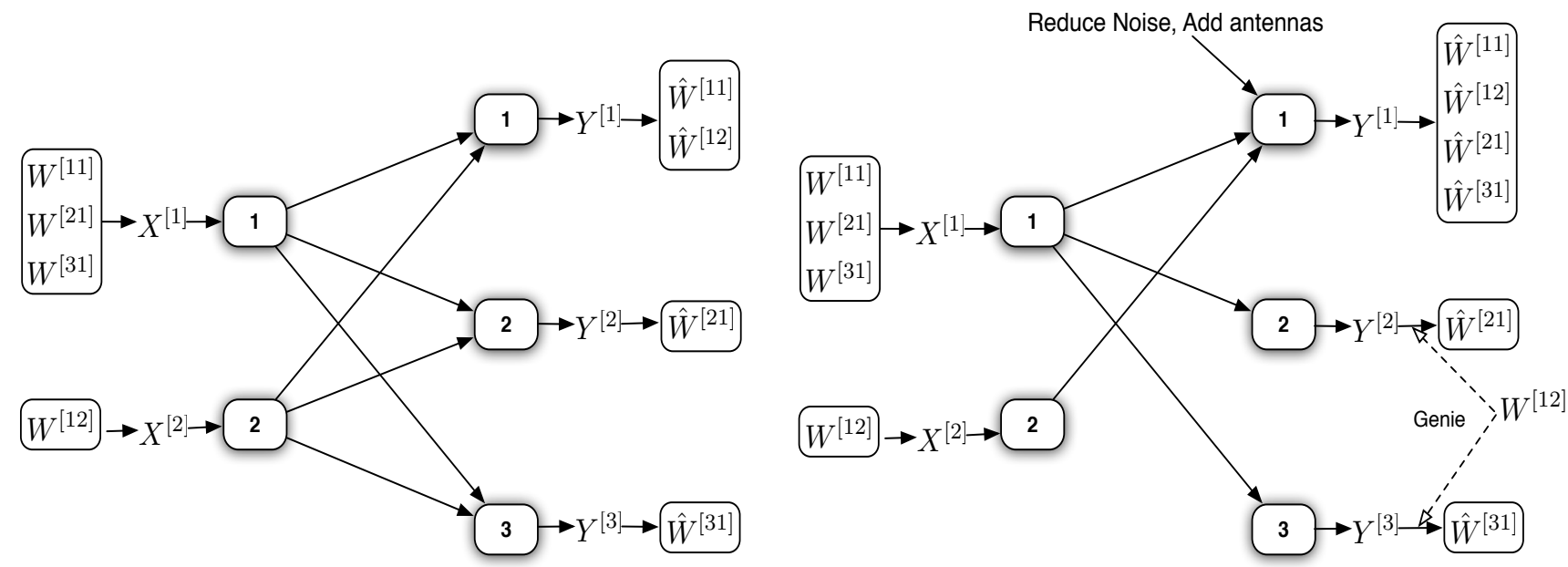

(a)

(b)

$$
W^{[32]}=W^{[22]}=\phi
$$

Fig. 3. (a) $2 \times 3 X$ channel with messages $\mathcal{W}^{11}$ (b) Converse argument in $2 \times 3 X$ channel for messages in $\mathcal{W}^{11}$. 
Consider any reliable coding scheme in the $X$ channel where all messages not in the set $\mathcal{W}^{n m}$ are eliminated. Now, suppose a genie provides all the messages $W^{[n q]}, q \in\{1,2, \cdots, m-1, m+1, m+2, \cdots, M\}$ to each of the receivers $1,2, \cdots, n-1, n+1, n+2 \cdots N$. Then, receivers $1,2,3, \ldots, n-1, n+1, \ldots, N$ can cancel the interference caused by $X^{[1]}, X^{[2]}, \cdots X^{[m-1]}, X^{[m+1]}, \ldots, X^{[M]}$ so that, effectively, the receiver $p$ obtains $\hat{Y}^{[p]}$ from the received signal where

$$
\hat{Y}^{[p]}=H^{[p m]} X^{[m]}+Z^{[p]}
$$

where $p \in\{1,2, \cdots, n-1, n+1, n+2, \cdots N\}$. Also using the coding scheme, receiver $n$ can decode its desired messages $W^{[n t]}, t=1,2,3, \ldots, M$. Therefore, receiver $n$ can subtract the effect of $X^{[1]}, X^{[2]}, \ldots, X^{[m-1]}, X^{[m+1]}, \ldots, X^{[M]}$ from the received signal so that it obtains $\hat{Y}^{[n]}$ where

$$
\hat{Y}^{[n]}=H^{[n m]} X^{[m]}+Z^{[n]}
$$

Notice that receivers $p \neq n$ are able to decode messages $W^{[p m]}$ from $\hat{Y}^{[p]}$. Now, we can reduce the noise at receiver $n$ and if $A_{n}^{r}<A_{m}^{t}$ we add antennas at receiver $n$ so that it has $\max \left(A_{m}^{t}, A_{n}^{r}\right)$ antennas. By reducing noise and adding antennas we can ensure that $\hat{Y}^{[p]}, p \neq n$ are degraded versions of $\hat{Y}^{[n]}$ (for the details of this argument in the multiple antenna case, see [7]). In other words, by reducing noise and possibly adding antennas, we can ensure that receiver $n$ can decode all messages $W^{[p m]}$. Note that the performance of the original coding scheme cannot deteriorate because of the genie or from reducing the noise or from adding antennas and therefore the converse argument is not affected. We have now shown that in a genie-aided channel with reduced noise (see Figure 3), receiver $m$ is able to decode all the messages in the set $\mathcal{W}^{n m}$ when these are the only messages present. This implies that degrees of freedom of the messages in the set $\mathcal{W}^{n m}$ lies within the degrees of freedom region of the multiple access channel with transmitters $1,2, \ldots, M$ and receiver $n$. Since receiver $n$ has $\max \left(A_{m}^{t}, A_{n}^{r}\right)$ antennas the total number of degrees of freedom for all messages in the set $\mathcal{W}^{n m}$ cannot be more than $\max \left(A_{m}^{t}, A_{n}^{r}\right)$. This gives us the outerbound

$$
\max _{\left[\left(d_{j i}\right)\right] \in \mathcal{D}} \sum_{q=1}^{N} d_{q m}+\sum_{p=1}^{M} d_{n p}-d_{n m} \leq \max \left(A_{m}^{t}, A_{n}^{r}\right)
$$

Repeating the arguments for each $m, n$ we arrive at the result of Theorem 1.

Since our focus in this paper is on the total degrees of freedom for the case when all nodes have one antenna, the following corollary establishes the needed outerbound.

Corollary 1: The total number of degrees of freedom of the $X$ channel with $M$ transmitters and $N$ receivers and 1 antenna at each node, is upper bounded by $\frac{M N}{M+N-1}$ i.e.

$$
\max _{\left[\left(d_{j i}\right)\right] \in \mathcal{D}} \sum_{n \in\{1,2, \ldots, N\}, m \in\{1,2, \ldots, M\}} d_{n m} \leq \frac{M N}{M+N-1}
$$

Proof: The bound can be obtained by summing all the $M N$ inequalities describing the outerbound of the degrees of freedom region and setting $A_{m}^{t}=A_{n}^{r}=1$ for all transmitters and receivers.

The outerbound of Theorem 1 is not only useful for the total number of degrees of freedom, but rather it bounds the entire degrees of freedom region of the $M \times N$ user $X$ network. In other words, Theorem 1 provides an outerbound for any fully connected distributed single hop network under the given system model. For example, consider a hypothetical channel with 3 single antenna transmitters and 3 single antenna receivers, and 6 messages, $W^{[j i]}, i \neq j, i, j \in\{1,2,3\}$, i.e, the $3 \times 3$ user $X$ channel with $W^{[11]}=W^{[22]}=W^{[33]}=\phi$. The solution to the 
following linear programming problem provides an outerbound for the total number of degrees of freedom of this channel.

$$
\begin{gathered}
\max _{\left[\left(d_{i j}\right)\right]} \sum_{m \neq l} d_{m l} \\
\text { s.t } \quad \sum_{q=1}^{3} d_{m q}+\sum_{p=1}^{3} d_{p l}-d_{m l} \leq 1 \quad \forall(m, l) \in\{1,2,3\} \times\{1,2,3\}
\end{gathered}
$$

In many cases of interest these outerbounds can be shown to be tight. For example, in the $2 \times 2$ user $X$ network, the outerbound of Theorem 1 is shown to represent the entire degrees of freedom region [15].

\section{Interference Alignment And Innerbounds on the Degrees of Freedom}

The following is the main result of this section.

Theorem 2: The $M \times N$ user $X$ network with single antenna nodes has $\frac{M N}{M+N-1}$ degrees of freedom.

The converse for the theorem is already proved in the corollary to Theorem 1.

The achievable scheme for the $X$ networks are based on interference alignment and zero-forcing. For the general $M \times N$ user $X$ network we provide a partial interference alignment based innerbound that approaches the outerbound as we increase the size of the supersymbols (channel extensions). While the degrees of freedom achieved by this scheme can come within $\epsilon$ of the degrees of freedom outerbound for any $\epsilon>0$, the two are never exactly equal. This is sufficient for a degree of freedom characterization, but it does not provide an $O(1)$ capacity characterization. In some cases (when either $M=2$ or $N=2$ ) we are able to create perfect interference alignment schemes so that the degrees of freedom outerbound is exactly achieved with a finite channel extension. In these cases, the degrees of freedom also leads to a capacity characterization that is accurate within $O(1)$.

Before we proceed to describe the achievable schemes, we present a corollary to Theorem 2.

Corollary 2: Let $D_{\Sigma}$ represent the total number of degrees of freedom of the $M \times N$ user $X$ network where all transmitting and receiving nodes have $A$ antennas each.Then,

$$
\frac{A M N}{M+N-1 / A} \leq D_{\Sigma} \leq \frac{A M N}{M+N-1}
$$

Proof: The degrees of freedom outerbound follows from Theorem 1. The innerbound can be derived using an achievable scheme that treats each antenna in the network as a single distributed user. So, effectively, for this achievable scheme, the network is a $A M \times A N$ user $X$ network with single-antenna nodes. Then, Theorem 2 implies that $\frac{A M N}{M+N-1 / A}$ degrees of freedom are achievable.

Note that our degrees of freedom characterization of the $M \times N$ user MIMO $X$ network is not tight, if $A>1$. We now proceed to describe the perfect interference alignment scheme for the $M \times 2$ user $X$ network. Two preliminary lemmas used in proofs presented in the subsequent sections of the paper are placed in Appendix I.

\section{A. Perfect Interference Alignment for the $M \times 2$ user $X$ network}

The outerbound for the $M \times 2$ user $X$ channel states that it cannot achieve more than a total of $\frac{2 M}{M+1}$ degrees of freedom. We now present the construction of an interference alignment scheme which achieves exactly $\frac{1}{M+1}$ degrees of freedom for each of the $2 M$ messages, thus exactly achieving the outerbound. The scheme we present here is an extension of the scheme presented in [15]. 
Consider a $M+1$ symbol extension of the channel formed by combining $M+1$ symbols into a super-symbol. This channel can be expressed as

$$
\begin{aligned}
& \mathbf{Y}^{[1]}(\kappa)=\sum_{m \in\{1,2, \ldots, M\}} \mathbf{H}^{[1 m]}(\kappa) \mathbf{X}^{[m]}(\kappa)+\mathbf{Z}^{[1]}(\kappa) \\
& \mathbf{Y}^{[2]}(\kappa)=\sum_{m \in\{1,2, \ldots, M\}} \mathbf{H}^{[2 m]}(\kappa) \mathbf{X}^{[m]}(\kappa)+\mathbf{Z}^{[2]}(\kappa)
\end{aligned}
$$

where $\mathbf{X}^{[m]}(\kappa)$ is a $(M+1) \times 1$ column vector representing the $M+1$ symbol extension of the transmitted symbol $X^{[m]}$, i.e

$$
\mathbf{X}^{[m]}(\kappa) \triangleq\left[\begin{array}{c}
X^{[m]}(\kappa(M+1)+1) \\
X^{[m]}(\kappa(M+1)+2) \\
\vdots \\
X^{[m]}((\kappa+1)(M+1))
\end{array}\right]
$$

Similarly $\mathbf{Y}^{[j]}$ and $\mathbf{Z}^{[j]}$ represent $M+1$ symbol extensions of the $Y^{[j]}$ and $Z^{[j]}$ respectively. $\mathbf{H}^{[j m]}$ is a diagonal $(M+1) \times(M+1)$ matrix representing the $M+1$ symbol extension of the channel, i.e.,

$$
\mathbf{H}^{[j m]}(\kappa) \triangleq\left[\begin{array}{cccc}
H^{[j m]}(\kappa(M+1)+1) & 0 & \cdots & 0 \\
0 & H^{[j m]}(\kappa(M+1)+2) & \cdots & 0 \\
\vdots & \cdots & \ddots & \vdots \\
0 & 0 & \cdots & H^{[j m]}((\kappa+1)(M+1))
\end{array}\right]
$$

We now describe an achievable scheme that achieves one degree of freedom $d_{j m}=1, j=1,2, m=1,2, \ldots, M$ for each message over this $M+1$ symbol extension, thus achieving a total of $2 M$ degrees of freedom over $M+1$ symbols.

The encoding strategy is as follows. Transmitter $m$ encodes messages $W^{[1 m]}$ and $W^{[2 m]}$ as two independent streams $x^{[1 m]}$ and $x^{[2 m]}$ and respectively transmits these two streams along directions $\mathbf{v}^{[1 m]}$ and $\mathbf{v}^{[2 m]}$ (See Figure 4 for the special case where $M=2$ ). We can then write

$$
\mathbf{X}^{[m]}=x^{[1 m]} \mathbf{v}^{[1 m]}+x^{[2 m]} \mathbf{v}^{[2 m]}
$$

The received message at receiver $j$ is

$$
\mathbf{Y}^{[j]}=\sum_{m=1}^{M} \mathbf{H}^{[j m]} x^{[1 m]} \mathbf{v}^{[1 m]}+\sum_{m=1}^{M} \mathbf{H}^{[j m]} x^{[2 m]} \mathbf{v}^{[2 m]}+\mathbf{Z}^{[j]}
$$

where $j=1,2$.

Receiver 1 decodes its $M$ desired messages by zero-forcing the all interference vectors $\mathbf{v}^{[2 m]}, m=1,2, \ldots, M$. In other words, receiver 1 first processes $\mathbf{Y}^{[1]}$ as $\tilde{\mathbf{Y}}^{[1]}=\mathbf{P}_{1} \mathbf{Y}^{[1]}$, where $\mathbf{P}_{1}$ is a matrix which represents the kernel (null-space) of the set of interfering column vectors, i.e., $\left[\mathbf{H}^{[11]} \mathbf{V}^{[21]} \mathbf{H}^{[12]} \mathbf{V}^{[22]} \ldots \mathbf{H}^{[1 M]} \mathbf{V}^{[2 M]}\right]$. Now to recover $M$ interference-free streams for desired signals from $\tilde{\mathbf{Y}}^{[1]}$, the matrix $\mathbf{P}_{1}$ has to have a dimension of $M$. In other words, the matrix $\left[\mathbf{H}^{[11]} \mathbf{V}^{[21]} \mathbf{H}^{[12]} \mathbf{V}^{[22]} \ldots \mathbf{H}^{[1 M]} \mathbf{V}^{[2 M]}\right]$ must have a dimension of 1 . Equivalently, all the interference streams must align along a common direction (See Figure 4). Therefore, vectors $\mathbf{v}^{[2 m]}, i=2, \ldots, M$ are picked so that their corresponding interference terms at receiver 1 perfectly align with the interference from transmitter 1 - i.e $\mathbf{H}^{[1 m]} \mathbf{v}^{[2 m]}$ lies along $\mathbf{H}^{[11]} \mathbf{v}^{[21]}$ for all $m=2, \ldots, M$.

$$
\mathbf{H}^{[1 m]} \mathbf{v}^{[2 m]}=\mathbf{H}^{[11]} \mathbf{v}^{[21]}, m=2, \ldots, M
$$




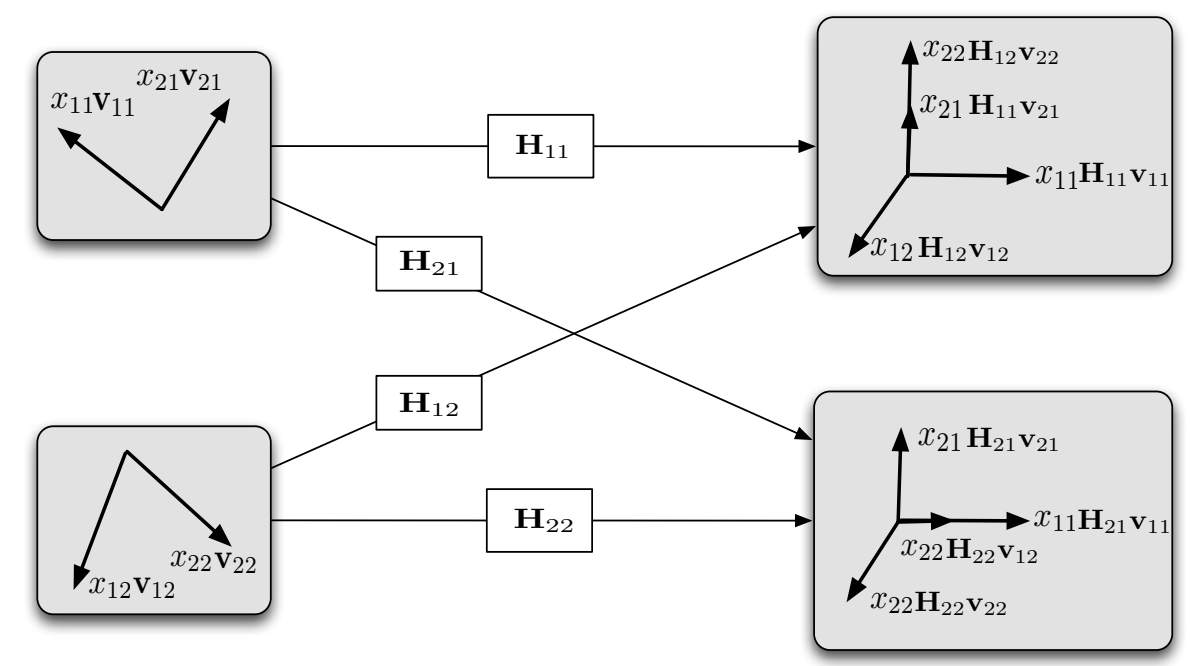

Fig. 4. Interference Alignment on the $2 \times 2$ user $X$ network [15]

This ensures that, from the point of view of receiver 1 , all the interference terms $\mathbf{H}^{[1 m]} \mathbf{v}[2 m]$ lie along a single vector $\mathbf{H}^{[11]} \mathbf{v}^{[21]}$, so that $\mathbf{P}_{1}$ has a dimension of $M$. Similarly, at receiver 2, we intend to decode its $M$ desired streams using $\tilde{\mathbf{Y}}^{[2]}=\mathbf{P}_{2} \mathbf{Y}^{[2]}$, where $\mathbf{P}_{2}$ is the kernel (null-space) of $\left[\mathbf{H}^{[21]} \mathbf{V}^{[11]} \mathbf{H}^{[22]} \mathbf{V}^{[12]} \ldots \mathbf{H}^{[2 M]} \mathbf{V}^{[1 M]}\right]$. To ensure that $\mathbf{P}_{2}$ has $M$ linearly independent columns, we pick $\mathbf{v}^{[1 m]}, m \neq 1$ as

$$
\mathbf{H}^{[2 m]} \mathbf{V}^{[1 m]}=\mathbf{H}^{[21]} \mathbf{V}^{[11]}, m=2 \ldots M
$$

Now that we have ensured all interference is restricted to only one dimension at each receiver, this dimension can be nulled to eliminate all interference, leaving $M$ interference free dimensions to recover the $M$ desired messages for each receiver. What is needed is that the desired signal vectors are linearly independent of the interference. In other words $\mathbf{P}_{1}$ (resp. $\mathbf{P}_{2}$ ), which is the null-space of $\mathbf{H}^{[11]} \mathbf{v}^{[21]}$ (resp. $\mathbf{H}^{[21]} \mathbf{v}^{[11]}$ ), should not null out any of the desired vectors at receiver 1 (resp. receiver 2 ). Therefore we need to pick $\mathbf{v}^{[11]}$ and $\mathbf{v}^{[21]}$ so that the following matrices are of full rank.

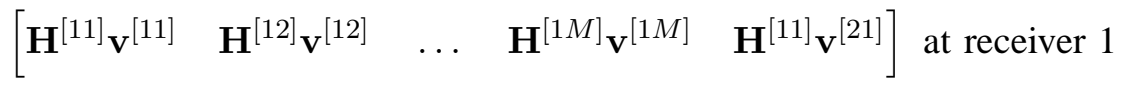

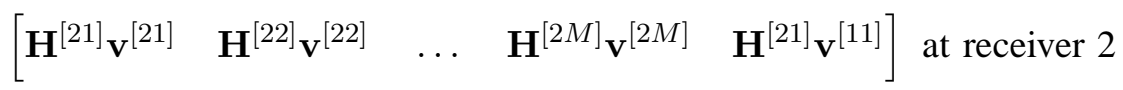

Note that the first $M$ column vectors of the above matrices represent the signal components, and the last column represents the aligned interference. We now pick the columns of $\mathbf{v}^{[11]}$ and $\mathbf{v}^{[21]}$ randomly from independent continuous distributions i.e.,

$$
\begin{array}{r}
\mathbf{v}^{[11]}=\left[\begin{array}{c}
p_{1} \\
p_{2} \\
\vdots \\
p_{M+1}
\end{array}\right] \\
\mathbf{v}^{[21]}=\left[\begin{array}{c}
q_{1} \\
q_{2} \\
\vdots \\
q_{M+1}
\end{array}\right]
\end{array}
$$


An important observation here is that once $\mathbf{v}^{[11]}$ and $\mathbf{v}^{[21]}$ are picked as above, equations (5) and (6) can be used to pick $\mathbf{v}^{[1 m]}, m \neq 1$ with just causal channel knowledge. i.e., the $l$ th component of the transmitted vector at any transmitter depends only on the first $l$ diagonal entries of $\mathbf{H}^{[j i]}$ (in fact, it depends only on the $l$ th diagonal entry). The desired signal can now be shown to be linearly independent of the interference at both receivers almost surely.

For example at receiver 1 , we need to show that matrix

$$
\boldsymbol{\Lambda}_{\mathbf{1}}=\left[\begin{array}{lllll}
\mathbf{H}^{[11]} \mathbf{V}^{[11]} & \mathbf{H}^{[12]} \mathbf{V}^{[12]} & \ldots & \mathbf{H}^{[1 M]} \mathbf{V}^{[1 M]} & \mathbf{H}^{[11]} \mathbf{V}^{[21]}
\end{array}\right]
$$

has full rank. Since all channel matrices are diagonal and full rank, we can multiply by $\left(\mathbf{H}^{[11]}\right)^{-1}$ and use equations (5) and (6) to replace the above matrix by

$$
\overline{\mathbf{\Lambda}}_{\mathbf{1}}=\left[\begin{array}{lllll}
\mathbf{v}^{[11]} & \left(\mathbf{H}^{[11]}\right)^{-1} \mathbf{H}^{[12]} \mathbf{H}^{[21]}\left(\mathbf{H}^{[22]}\right)^{-1} \mathbf{v}^{[11]} \quad \ldots \quad\left(\mathbf{H}^{[11]}\right)^{-1} \mathbf{H}^{[1 K]} \mathbf{H}^{[21]}\left(\mathbf{H}^{[2 K]}\right)^{-1} \mathbf{v}^{[11]} \quad \mathbf{v}^{[21]}
\end{array}\right]
$$

Let $H_{k}^{[j i]}$ is the $k$ th diagonal entry of $\mathbf{H}^{[j i]}$. We now make the following observations

1) The assumption on the channel model implies that $H_{k}^{[j i]} \mid\left\{H_{k^{\prime}}^{\left[j^{\prime} i^{\prime}\right]},\left(k^{\prime}, i^{\prime}, j^{\prime}\right) \neq(k, i, j)\right\}$ has a continuous (cumulative) distribution,

2) In the $k$ th row, the exponent of $H_{k}^{[1 m]}$ is 1 in the element in the $m$ th column of $\bar{\Lambda}_{\mathbf{1}}$ and 0 in all other columns for $m=2, \ldots, M$. Therefore, in the $k$ th row, $H_{k}^{[1 m]}$ has a different exponent in the element in the $m$ column, as compared to its exponent in the $m^{\prime}$ th column, for any $m \neq m^{\prime}$.

The above observations combined with the result of Lemma 1 presented in Appendix I imply that the matrix $\bar{\Lambda}_{1}$ has full rank of $M+1$ almost surely. Thus, the desired signal vectors are linearly independent of the aligned interference vectors almost surely.

Similarly, the desired signal can be shown to be linearly independent of the interference at receiver 2 almost surely. Therefore, $2 M$ independent streams are achievable over the $(M+1)$ symbol extension of the channel resulting in $\frac{2 M}{M+1}$ degrees of freedom over the original channel. Also, since the achievable scheme essentially creates $2 M$ point-to-point links over a $M+1$ symbol extension of the channel, it provides an $\mathcal{O}(1)$ capacity characterization [16] of the $M \times 2$ user $X$ network as

$$
C(\rho)=\frac{2 M}{M+1} \log (\rho)+\mathcal{O}(1)
$$

where $C(\rho)$ is the sum-capacity of the network as a function of transmit power $\rho$.

\section{B. Achievability for $2 \times M X$ network - Reciprocity of beamforming and zero-forcing based schemes}

Consider an $M \times N$ user $X$ network. We refer to this as the primal network. Consider any achievable scheme on this channel based on beamforming and zero-forcing. Specifically, consider any achievable scheme whose coding strategy maybe described as follows.

- Encoding - Transmitter $i$ encodes a message to receiver $j$ along independent streams and beamforms these streams along linearly independent vectors. For example, the $k$ th stream to receiver $j$ is encoded at transmitter $i$ as $x_{k}^{[j i]}$ and beamformed along direction $\mathbf{v}_{k}^{[j i]}$ as $x_{k}^{[j i]} \mathbf{v}_{k}^{[j i]}$.

- Decoding - Receiver $j$ decodes all the desired message streams through zero-forcing. For example, to decode the $k$ th stream from transmitter $i$, i.e, $x_{k}^{[j i]}$, the receiver projects the received vector along $\mathbf{u}_{k}^{[j i]}$ which nulls all undesired streams. 
The reciprocal (or dual) network is the network formed when the transmitters and receivers of the primal network are interchanged and the channel gains remain the same. Therefore, the dual of an $M \times N$ user $X$ network is a $N \times M$ user $X$ network. The channel gain between transmitter $i$ and receiver $j$ in the primal network is equal to the channel gain between transmitter $j$ and receiver $i$ in the dual network. It can be shown that corresponding to every zero-forcing based achievable scheme in the primal network, there exists a zero-forcing based achievable scheme in the reciprocal network that achieves the same number of degrees of freedom as the primal network. In particular, the coding scheme that achieves this in the dual network may be described as follows.

- Encoding - In the dual network, transmitter $j$ encodes a message to receiver $i$ along linearly independent streams and beamforms these streams along directions that were used for zero-forcing in the primal network. For example, the $k$ th stream to receiver $i$ is encoded as $\bar{x}_{k}^{[i j]}$ and beamformed along direction $\mathbf{u}_{k}^{[j i]}$ as $\bar{x}_{k}^{[i j]} \mathbf{u}_{k}^{[j i]}$, (where $\mathbf{u}_{k}^{[j i]}$ represents the zero-forcing vector used in the primal network by receiver $j$ to decode the $k$ th stream from transmitter $i$ )

- Decoding - Receiver $i$ decodes all the desired streams through zero-forcing along directions that were the beamforming directions in the primal network. For example, in the dual network, to decode the $k$ th desired stream from transmitter $j$ i.e $\bar{x}_{k}^{[i j]}$, receiver $i$ projects the received vector along vector $\mathbf{v}_{k}^{[j i]}-$ where $\mathbf{v}_{k}^{[j i]}$ represents the beamforming vector used in the primal network by transmitter $i$ to transmit the $k$ th stream to receiver $j$

It can be easily verified that the above scheme maps every independent interference-free stream in the primal $M \times N$ user $X$ network to an independent interference-free stream in the dual $N \times M$ user $X$ network and thus achieves the same number of degrees of freedom in the dual network. This scheme therefore establishes a general duality of beamforming and zero-forcing based interference alignment schemes. Below, we provide a formal proof of reciprocity for the $M=2$ case. The proof serves as an achievable scheme in the $2 \times N$ user $X$ network with frequency selective channels.

Proof of Theorem 2 for the $2 \times N$ user $X$ network: The $2 \times N$ user $X$ network maybe represented as

$$
Y^{[m]}(\kappa)=H^{[m 1]}(\kappa) X^{[1]}(\kappa)+H^{[m 2]}(\kappa) X^{[2]}(\kappa)+Z^{[m]}(\kappa), m=1,2, \ldots, N
$$

Consider the $N+1$ symbol extension of the $2 \times N$ user $X$ network which can be represented as

$$
\mathbf{Y}^{[m]}(\kappa)=\sum_{l \in\{1,2\}} \mathbf{H}^{[m l]}(\kappa) \mathbf{X}^{[l]}(\kappa)+\mathbf{Z}^{[m]}(\kappa), m=1,2 \ldots N
$$

The vectors $\mathbf{X}^{[i]}, \mathbf{Y}^{[i]}, \mathbf{Z}^{[i]}$ are $(N+1) \times 1$ vectors and $\mathbf{H}^{[i j]}$ is $(N+1) \times(N+1)$ matrix (Refer to (3), (4) for a similar channel extension over the $M \times 2$ user $X$ network) The reciprocal of this extended network is a $N \times 2$ user $X$ network which can be expressed as

$$
\begin{aligned}
& \overline{\mathbf{Y}}^{[1]}(\kappa)=\sum_{m \in\{1,2 \ldots N\}} \overline{\mathbf{H}}^{[1 m]}(\kappa) \overline{\mathbf{X}}^{[m]}(\kappa)+\overline{\mathbf{Z}}^{[1]}(\kappa) \\
& \overline{\mathbf{Y}}^{[2]}(\kappa)=\sum_{m \in\{1,2 \ldots N\}} \overline{\mathbf{H}}^{[2 m]}(\kappa) \overline{\mathbf{X}}^{[m]}(\kappa)+\overline{\mathbf{Z}}^{[2]}(\kappa)
\end{aligned}
$$

where the over-bar notation indicates quantities in the reciprocal channel. The vectors $\overline{\mathbf{X}}^{[i]}, \overline{\mathbf{Y}}^{[i]}, \overline{\mathbf{Z}}^{[i]}$ are $(N+1) \times 1$ vectors and $\overline{\mathbf{H}}^{[i j]}$ is $(N+1) \times(N+1)$ matrix. Note that in the reciprocal channel, the channel gains are identical to those in the original channel, and we can write

$$
\overline{\mathbf{H}}^{[i j]}(\kappa)=\mathbf{H}^{[j i]}(\kappa), j \in\{1,2 \ldots N\}, i \in\{1,2\}
$$


Now, consider the achievable scheme over this $N \times 2$ user $X$ channel, as described in Section V-A. In the achievable scheme, transmitter $i$ encodes message $\bar{W}^{[j i]}$ as $\bar{x}^{[j i]}$ and beamforms it along direction $\overline{\mathbf{v}}^{[j i]}$ so that the signal at receiver $j$, for $j \in\{1,2\}$, is

$$
\overline{\mathbf{Y}}^{[j]}=\sum_{m=1}^{N} \bar{x}^{[1 m]} \overline{\mathbf{H}}^{[j m]} \overline{\mathbf{v}}^{[1 m]}+\sum_{m=1}^{N} \bar{x}^{[2 m]} \overline{\mathbf{H}}^{[j m]} \overline{\mathbf{v}}^{[2 m]}+\overline{\mathbf{Z}}^{[j]}
$$

The dependence on the index $\kappa$ is dropped above for compactness. In this achievable scheme, receiver $j$ decodes $\bar{x}^{[j i]}$ using zero-forcing. Let $\overline{\mathbf{u}}^{[j i]}$ represent the zero-forcing vector used by receiver $j$ to decode $\bar{x}^{[j i]}$. Since $\overline{\mathbf{u}}^{[j i]}$ nulls all the interfering streams, we can write

$$
\begin{gathered}
\left(\overline{\mathbf{u}}^{[j i]}\right)^{T} \overline{\mathbf{H}}^{[j m]} \overline{\mathbf{v}}^{[l m]}=0, \forall(l, m) \neq(j, i) \\
\Rightarrow\left(\overline{\mathbf{u}}^{[j i]}\right)^{T} \overline{\mathbf{Y}}^{[j]}=\bar{x}^{[j i]}\left(\overline{\mathbf{u}}^{[j i]}\right)^{T} \overline{\mathbf{H}}^{[j i]} \overline{\mathbf{v}}^{[j i]}+\left(\overline{\mathbf{u}}^{[j i]}\right)^{T} \overline{\mathbf{Z}}^{[j]}
\end{gathered}
$$

We now use the above fact to construct beamforming directions $\mathbf{v}^{[i j]}$ and zero-forcing vectors $\mathbf{u}^{[i j]}$ in the original $2 \times N$ user $X$ network. The beamforming and zero-forcing vectors we construct will enable receiver $i$ to decode message $W^{[i j]}$ by nulling interference from all other vectors. In the primal network, let transmitter $j$ encode message $W^{[i j]}$ to receiver $i$ as $x^{[i j]}$ where $j \in\{1,2\}, i \in\{1,2, \ldots N\}$. The beamforming directions of the primal network are chosen to be the zero-forcing vectors in the dual channel, i.e., $\mathbf{v}^{[i j]}=\overline{\mathbf{u}}^{[j i]}, j \in\{1,2\}, i \in\{1,2, \ldots N\}$. The transmitted message is therefore

$$
\mathbf{X}^{[j]}=\sum_{m=1}^{N} x^{[m j]} \mathbf{v}^{[m j]}=\sum_{m=1}^{N} x^{[m j]} \overline{\mathbf{u}}^{[j m]}, j=1,2
$$

The received vector at receiver $i$ is

$$
\begin{aligned}
\mathbf{Y}^{[i]} & =\sum_{m=1}^{N}\left(x^{[m 1]} \mathbf{H}^{[i 1]} \mathbf{v}^{[m 1]}+x^{[m 2]} \mathbf{H}^{[i 2]} \mathbf{v}^{[m 2]}\right)+\mathbf{Z}^{[i]} \\
& =\sum_{m=1}^{M}\left(x^{[m 1]} \mathbf{H}^{[i 1]} \overline{\mathbf{u}}^{[1 m]}+x^{[m 2]} \mathbf{H}^{[i 2]} \overline{\mathbf{u}}^{[2 m]}\right)+\mathbf{Z}^{[i]}
\end{aligned}
$$

for $i \in\{1,2, \ldots, N\}$. Now, at receiver $i$, stream $x^{[i j]}$ is decoded by projecting the received vector along $\mathbf{u}^{[i j]}=\overline{\mathbf{v}}^{[j i]}$, i.e., the vector that was used for beamforming at transmitter $i$ in the dual network.

$$
\begin{aligned}
\left(\overline{\mathbf{v}}^{[j i]}\right)^{T} \mathbf{Y}^{[i]} & =\sum_{m=1}^{M}\left(\overline{\mathbf{v}}^{[j i]}\right)^{T}\left(x^{[m 1]} \mathbf{H}^{[i 1]} \overline{\mathbf{u}}^{[1 m]}+x^{[m 2]} \mathbf{H}^{[i 2]} \overline{\mathbf{u}}^{[2 m]}\right)+\left(\overline{\mathbf{v}}^{[j i]}\right)^{T} \mathbf{Z}^{[i]} \\
& =\sum_{m=1}^{M}\left(x^{[m 1]}\left(\overline{\mathbf{v}}^{[j i]}\right)^{T} \mathbf{H}^{[i 1]} \overline{\mathbf{u}}^{[1 m]}+x^{[m 2]}\left(\overline{\mathbf{v}}^{[j i]}\right)^{T} \mathbf{H}^{[i 2]} \overline{\mathbf{u}}^{[2 m]}\right)+\left(\overline{\mathbf{v}}^{[j i]}\right)^{T} \mathbf{Z}^{[i]} \\
& =\sum_{m=1}^{M}\left(x^{[m 1]}\left(\mathbf{H}^{[i 1]} \overline{\mathbf{u}}^{[1 m]}\right)^{T} \overline{\mathbf{v}}^{[j i]}+x^{[m 2]}\left(\mathbf{H}^{[i 2]} \overline{\mathbf{u}}^{[2 m]}\right)^{T} \overline{\mathbf{v}}^{[j i]}\right)+\left(\overline{\mathbf{v}}^{[j i]}\right)^{T} \mathbf{Z}^{[i]} \\
& =\sum_{m=1}^{M}\left(x^{[m 1]}\left(\overline{\mathbf{u}}^{[1 m]}\right)^{T} \overline{\mathbf{H}}^{[1 i]} \overline{\mathbf{v}}^{[j i]}+x^{[m 2]}\left(\overline{\mathbf{u}}^{[2 m]}\right)^{T} \overline{\mathbf{H}}^{[2 i]} \overline{\mathbf{v}}^{[j i]}\right)+\left(\overline{\mathbf{v}}^{[j i]}\right)^{T} \mathbf{Z}^{[i]}, j=1,2, i=1,2, \ldots, N
\end{aligned}
$$

Above, we have used equation (8) in the final step. Combining equation (9) with the above equation, we get

$$
\left(\overline{\mathbf{v}}^{[j i]}\right)^{T} \mathbf{Y}^{[i]}=x^{[i j]}\left(\overline{\mathbf{u}}^{[j i]}\right)^{T} \overline{\mathbf{H}}^{[j i]} \overline{\mathbf{v}}^{[j i]}+\left(\overline{\mathbf{v}}^{[j i]}\right)^{T} \mathbf{Z}^{[i]}
$$


Thus, the zero-forcing vector $\overline{\mathbf{v}}^{[j i]}$ cancels all interference and $x^{[i j]}$ can be decoded free of interference. This implies that 1 degree of freedom is achieved for message $W^{[i j]}$, for each $i \in\{1,2 \ldots N\}, j \in\{1,2\} .2 N$ degrees of freedom are achieved over the extended $2 \times N$ user $X$ network implying that $\frac{2 N}{N+1}$ degrees of freedom are achieved in the original $2 \times N$ user $X$ network. The reciprocal scheme also implies that the $O(1)$ capacity of the $2 \times N$ user $X$ network is

$$
C(\rho)=\frac{2 N}{N+1} \log (\rho)+\mathcal{O}(1)
$$

where $C(\rho)$ is the sum-capacity of the network as a function of transmit power $\rho$

Remark: The achievable scheme described in above proof requires the nodes to have apriori channel knowledge of all the channel co-efficients. This is because, to construct beamforming vectors in the $2 \times N$ user $X$ network, the transmitters need to be aware of the zero-forcing vectors in the dual $N \times 2$ user $X$ network. Construction of zero-forcing vectors at the receivers in the dual $N \times 2$ user $X$ network requires knowledge of all the channel co-efficients during the transmission. Therefore, the encoding strategy based on reciprocity requires non-causal channel knowledge, if $\kappa$ indicates time-index in (7). Therefore, from a practical perspective, the above achievable scheme may be feasible when $\kappa$ represents frequency-index, and channels are frequency-selective. This is because, if $\kappa$ represents frequency, the achievable scheme only requires nodes to have knowledge of the channels over all frequencies and therefore does not violate any causality constraints. It must be noted that the Theorem 2 holds whether $\kappa$ represents time, or frequency. The partial interference alignment scheme we describe in the next subsection is applicable in the $2 \times N$ user $X$ network even if channels only have causal channel knowledge, and $\kappa$ represents time-index.

\section{Partial Interference Alignment for General $M \times N$ user $X$ Networks}

The solution for the general case does not immediately follow from the solution to the $M \times 2$ user $X$ network. To see this, consider the $3 \times 3$ user $X$ network, where we intend to achieve the outerbound of $\frac{9}{5}$ degrees of freedom. Consider a 5 symbol extension of the channel. Over this extended channel, consider a hypothetical achievable scheme where each of the 9 messages achieves 1 degree of freedom if possible, using beamforming at all transmitters and zero-forcing at all receivers. Let message $W^{[i j]}$ be beamformed along vector $\mathbf{v}^{[i j]}$ at transmitter $j$. Receiver $i$ intends to decode $W^{[i j]}$ using zero-forcing. At receiver $i$, to decode 3 independent messages $W^{[i 1]}, W^{[i 2]}, W^{[i 3]}$ using zeroforcing, the vectors corresponding to the desired messages occupy 3 linearly independent directions. Since signals come from a space of dimension 5, the 6 interfering vectors must occupy the remaining 2 dimensions. Although there appears to be no obvious generalization of the $3 \times 2$ network to achieve this, one possible technique maybe the following.

1) At receiver 1 , the vectors $\mathbf{v}^{[2 j]}, j=1,2,3$ which correspond to receiver 2 align a long a common direction, i.e.,

$$
\mathbf{H}^{[11]} \mathbf{V}^{[21]}=\mathbf{H}^{[12]} \mathbf{V}^{[22]}=\mathbf{H}^{[13]} \mathbf{V}^{[23]}
$$

Similarly, the vectors corresponding to receiver 3 , i.e. $\mathbf{v}^{[3 j]}, j=1,2,3$, align along a different common direction, so that

$$
\mathbf{H}^{[11]} \mathbf{V}^{[31]}=\mathbf{H}^{[12]} \mathbf{V}^{[32]}=\mathbf{H}^{[13]} \mathbf{V}^{[33]}
$$

Thus, the total dimension of the interference is 2 and receiver 1 can decode all its desired messages. 
2) At receiver 2, interference vectors corresponding to receiver 1 align along a common direction, and interference vectors corresponding to receiver 3 align along a different common direction.

3) At receiver 3 , interference vectors corresponding to receiver 2 align along a common direction, and interference vectors corresponding to receiver 1 align along a different common direction.

Along with the above conditions, we need the desired signal vectors to be linearly independent of the 2 interference dimensions at each receiver. Note that if we can find vectors $\mathbf{v}^{[i j]}$ that satisfy the conditions listed above along with the linear independence condition, then that would show achievability of $\frac{9}{5}$ degrees of freedom using a 5 symbol extension. However, we argue below that construction of $\mathbf{v}^{[i j]}$ satisfying all these conditions is infeasible. Note that, in the construction just described, the beamforming vectors corresponding to receiver 2 align at receivers 1 and 3 , i.e.

$$
\mathbf{H}^{[i 1]} \mathbf{V}^{[21]}=\mathbf{H}^{[i 2]} \mathbf{V}^{[22]}=\mathbf{H}^{[i 3]} \mathbf{V}^{[23]}, i=1,3
$$

(12) implies that

$$
\begin{aligned}
\mathbf{v}^{[22]} & =\left(\mathbf{H}^{[12]}\right)^{-1} \mathbf{H}^{[11]} \mathbf{v}^{[21]} \\
& =\left(\mathbf{H}^{[32]}\right)^{-1} \mathbf{H}^{[31]} \mathbf{v}^{[21]} \\
\Rightarrow \mathbf{v} & =\left(\mathbf{H}^{[11]}\right)^{-1} \mathbf{H}^{[12]}\left(\mathbf{H}^{[32]}\right)^{-1} \mathbf{H}^{[31]} \mathbf{V}^{[21]}
\end{aligned}
$$

In other words, $\mathbf{v}^{[21]}$ is an eigen-vector of $\mathbf{T} \triangleq\left(\mathbf{H}^{[11]}\right)^{-1} \mathbf{H}^{[12]}\left(\mathbf{H}^{[32]}\right)^{-1} \mathbf{H}^{[31]}$. Since $\mathbf{T}$ is diagonal, this implies that $\mathbf{v}^{[21]}$ is a column vector of the identity matrix. This means that $\mathbf{v}^{[21]}$ is an eigen vector of all channel matrices, since they are all diagonal. Further, equation (12) implies that $\mathbf{v}^{[22]}$ is merely a scaled version of $\mathbf{v}^{[21]}$. This implies that at receiver $2, \mathbf{H}^{[21]} \mathbf{v}^{[21]}$ and $\mathbf{H}^{[22]} \mathbf{v}^{[22]}$ are linearly dependent, since they both are scaled versions of the same column of the identity matrix, i.e., we can write

$$
\operatorname{span}\left(\mathbf{H}^{[22]} \mathbf{v}^{[22]}\right)=\operatorname{span}\left(\mathbf{H}^{[21]} \mathbf{v}^{[21]}\right)=\operatorname{span}\left(\mathbf{v}^{[21]}\right)
$$

Therefore, the desired signals are not decodable at receiver 2 using zero-forcing of interference. This implies that the scheme described in Section V-A cannot be generalized in the manner described above. However, we note that this argument does not preclude the existence of a general zero-forcing based interference alignment scheme that uses a finite symbol extension of the channel to achieve the outerbound. We only claim that the construction described through alignment conditions 1),2) and 3) listed above is not possible. The main result of this work is that the degrees of freedom outerbound of Theorem 1 is tight, and despite the apparent complexity of the $X$ channel, interference alignment is indeed possible. We now proceed to provide a brief intuitive overview of our achievable scheme. A formal proof is presented in Appendix II.

Consider any arbitrary $n>0, n \in \mathbb{N}$. The achievable scheme we provide considers a $(M+N-1)(f(n)+o(f(n)))$ symbol extension of the channel, where

$$
f(n) \triangleq n^{(M-1)(N-1)}
$$

Over this extended channel, we construct an achievable scheme so that each message achieves $f(n)+o(f(n))$ degrees of freedom. By picking an arbitrarily large $n$ we can achieve, on the original (i.e., not extended) channel, arbitrarily close to $\frac{1}{M+N-1}$ degrees of freedom for each message, per orthogonal time/frequency dimension. Hence, as $n \rightarrow \infty$, the achieved number of degrees of freedom is arbitrarily close to $\frac{M N}{M+N-1}$ (See Figure 6). The achievable scheme over the extended channel uses beamforming at all transmitters and zero-forcing at all receivers. Specifically, 


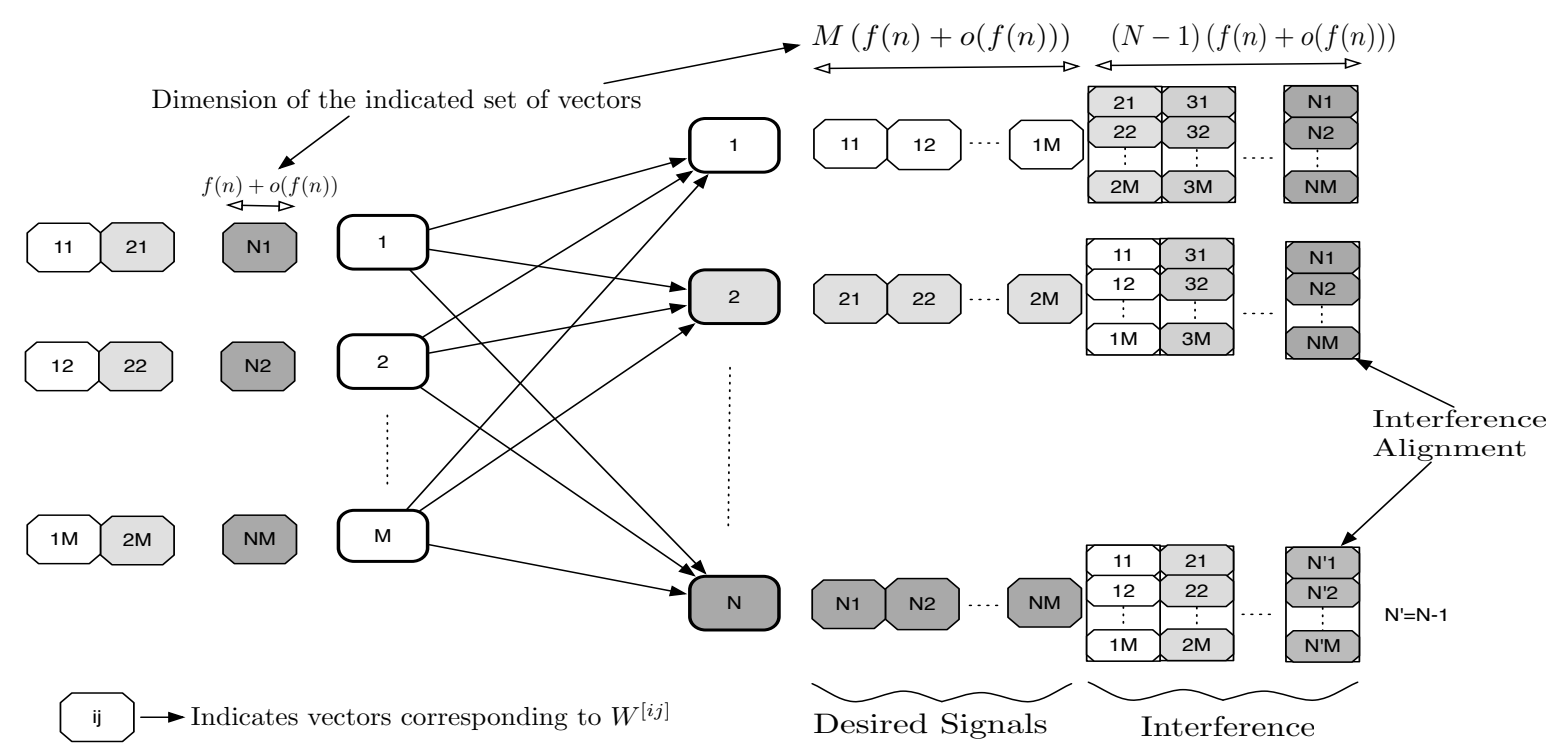

Fig. 5. Interference Alignment on the $M \times N$ user $X$ network

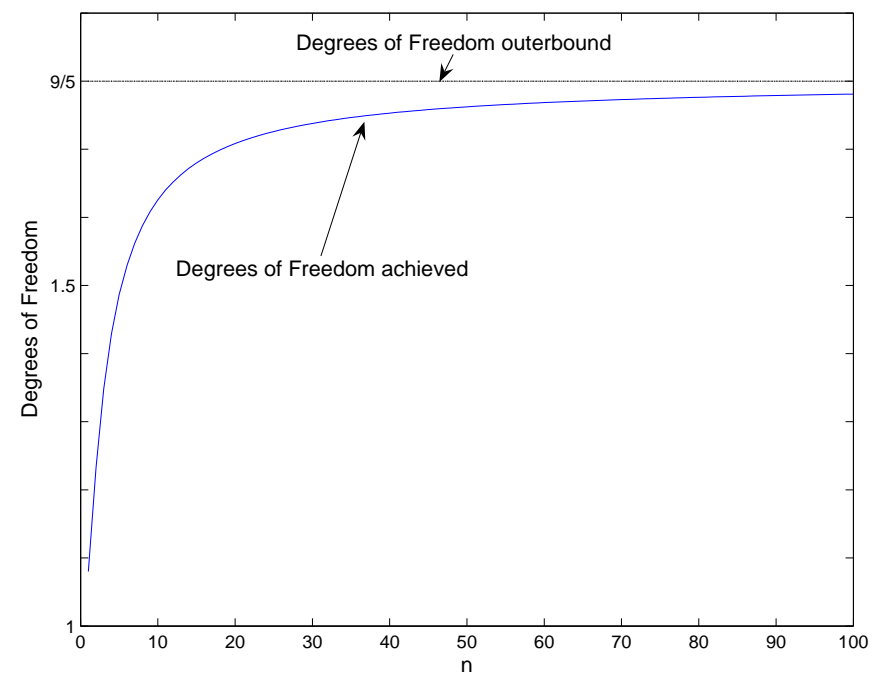

Fig. 6. The number of degrees of freedom achieved versus parameter $n$ for the $3 \times 3$ user $X$ network

message $W^{[i j]}$ is beamformed along $f(n)+o(f(n))$ linearly independent directions at transmitter $j$. Receiver $i$ correspondingly decodes the message by projecting the received vector into a space of dimension $f(n)+o(f(n))$ which nulls the interference. The beamforming and zero-forcing directions are chosen as depicted in Figure 5. As shown in the figure, at receiver $j$, the $f(n)+o(f(n))$ beams corresponding to $W^{[i k]}, i \neq j$ align with the $f(n)+o(f(n))$ beams corresponding to $W^{[i l]}, k \neq l$. In other words, at receiver $j \neq i$, the union of the received vectors corresponding to messages $W^{[i 1]}, W^{[i 2]}, \ldots W^{[i M]}$ lies in a space of dimension $f(n)+o(f(n))$. Note that for a fixed $n$, the overlap between these vectors is partial, i.e. the vectors corresponding to $W^{[i 1]}$ do not perfectly co-incide with the vectors corresponding to $W^{[i 2]}$ at receiver $j \neq i$. However, number of vectors that do not align is captured by the $o(f(n))$ factor, which is negligible to $f(n)$ - the portion that aligns - if $n$ is large. In other words, the alignment is asymptotically perfect as $n$ becomes arbitrarily large. Since the cardinality 


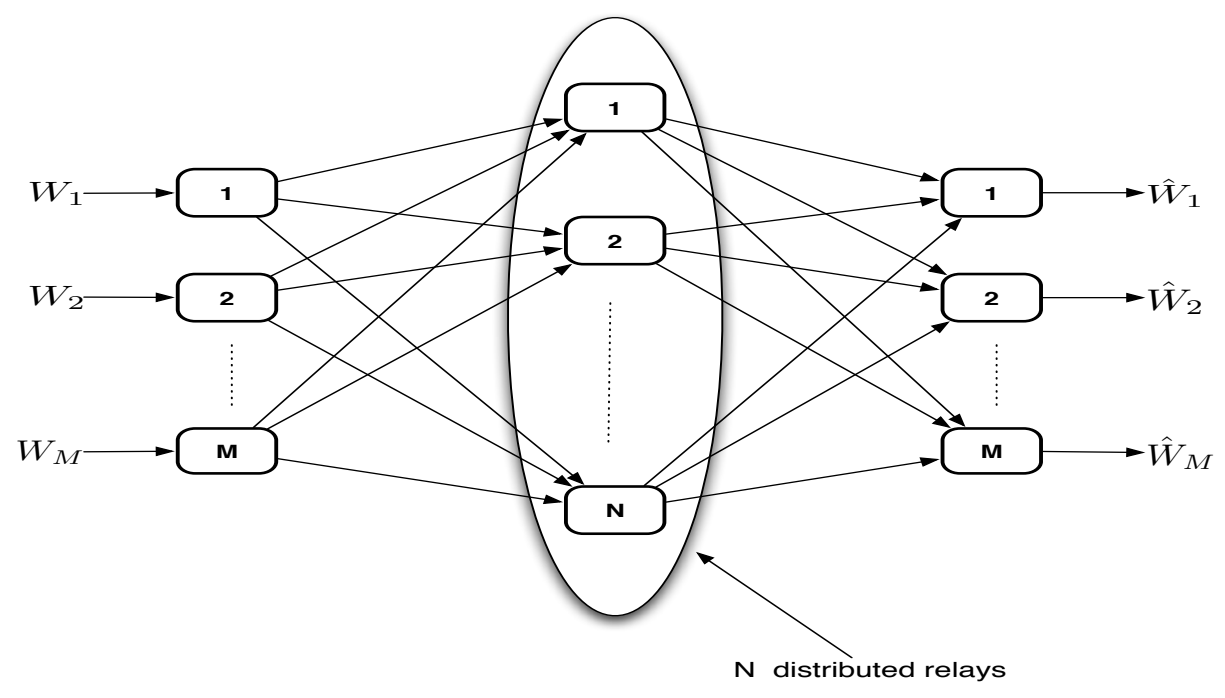

Fig. 7. The parallel relay network

of the set $\{i \neq j, i \in\{1,2 \ldots N\}\}$ is $N-1$, the dimension of all the interfering vectors at receiver $j$ is $(N-$ 1) $(f(n)+o(f(n)))$. Also, since corresponding to each of the $M$ desired messages, the number of beams received is $f(n)+o(f(n))$, the dimension of the set of vectors corresponding to the desired messages is $M(f(n)+o(f(n)))$. If the set of interfering vectors at a receiver are linearly independent of the set of desired vectors, then, in a space of dimension $(M+N-1)(f(n)+o(f(n)))$, the $M(f(n)+o(f(n)))$ desired streams can be decoded by nulling the $(N-1)(f(n)+o(f(n)))$ interfering dimensions. We show that this linear independence condition can be satisfied at all receivers. The precise construction of beamforming and zero-forcing vectors that leads to this can be found in the proof placed in Appendix II.

\section{Degrees of Freedom of the Parallel Relay Network}

In this section, we present an application of the results of the previous section. We provide an alternate proof of the degrees of freedom characterization of the parallel relay network (Figure 7), first studied in [8].

Consider a two hop parallel relay network with $M$ distributed single antenna transmitters and $M$ distributed single antenna receivers. We assume that the intermediate hop has $N$ half-duplex relays.

Much like the $M$ user interference network, transmitter $j$ has a single message $W^{[j]}$ to transmit to receiver $j$, where $j=1,2, \ldots M$ and thus there are a total of $M$ messages in this channel. All transmitters and relays have an average power constraint of $\rho$. Through an achievable scheme based on amplify-and-forward strategy at the relays, [8] shows that this network has $M / 2$ degrees of freedom if the number of relays $N$ approaches infinity. We use the degrees of freedom characterization of $X$ network to provide an alternate optimal achievable scheme to show the same result by treating this network as a concatenation of two $X$ networks. Notice that the interpretation of the parallel relay network as a compound of two $X$ networks results is restrictive, since it implies that the relays are forced to decode, and hence fixes coding strategies to decode-and-forward based achievable schemes. However, in time-varying (or frequency-selective) channels, this scheme is optimal, as it achieves $M / 2$ degrees of freedom.

Theorem 3: $\frac{M N}{2(M+N-1)}$ degrees of freedom are achievable by a decode-and-forward strategy in the two-hop parallel relay network with $M$ distributed transmitters and receivers with $N$ distributed half-duplex relays. If $N \rightarrow \infty$, this parallel relay network has $M / 2$ degree of freedom. 
The proof follows from the degrees of characterization of the $X$ channel. For brevity's sake, we only provide an outline of the proof here. The message $W^{[j]}$ is split into $N$ independent sub-messages $W_{n}^{[j]}, n=1,2 \ldots N$ with message $W_{k}^{[j]}$ meant to be decoded by relay $k$. Our achievable scheme operates in two phases, both of which are active for half the duration of transmission. Let the block length of whole transmission be $2 T$ symbols. In the first phase corresponding to the first $T$ symbols, the coding scheme corresponding to the $M \times N$ user $X$ network is employed so that $\frac{T}{M+N-1} \log (\rho)+o(\log (\rho))$ bits corresponding to each sub-message is transmitted. For the second phase, notice that each relay has $\frac{T}{M+N-1} \log (\rho)+o(\log (\rho))$ bits of information for each receiver - these bits are transmitted in $T$ symbols over the $N \times M$ user $X$ network to the receivers. Since there is a total of $M N$ submessages in the system, a total of $\frac{T M N}{M+N-1} \log (\rho)+o(\log (\rho))$ bits are transmitted over the network in $2 T$ symbols thus achieving $\frac{M N}{2(M+N-1)}$ degrees of freedom per symbol overall.

\section{CONCLUSION}

The $X$ network is arguably the most important single-hop network since it contains, within itself, most other one-way fully connected single hop networks. For instance, the 2 user MAC, BC and interference channels are all embedded in a two user $X$ channel, and therefore can be derived by setting appropriate messages to null. We provide an outerbound for the degrees of freedom region of the $\mathrm{X}$ network with arbitrary number of single-antenna transmitters and receivers and no shared information among nodes. We also show that the total number of degrees of freedom of the $M \times N$ user $X$ network is equal to $\frac{M N}{M+N-1}$. The degrees of freedom region outerbound is very useful since it can be used to bound the number of degrees of freedom of most practical distributed single-hop wireless ad-hoc networks.

This is an optimistic result from the point of view of network information theory. It suggests that, from a degrees of freedom perspective, distributed single antenna nodes with no prior common information can behave as a single node with multiple antennas if they are transmitting to or receiving from a relatively large number of nodes. We provide an example of this scenario in the form of a degrees of freedom of the parallel relay network.

The result of this work demonstrates the power of the technique of interference alignment combined with zeroforcing. The optimality of interference alignment in the $X$ network motivates a closer look at interference alignment based schemes. For example, we note that the optimal achievable scheme uses arbitrarily long channel extensions in most cases. From a practical perspective, an important extension of this work is the study of the performance of achievable schemes restricted to fixed finite channel extensions. It has been observed that arbitrarily long channel extensions can be avoided without compromising optimality in the 3 user interference network if all the nodes are equipped with multiple antennas. A study of the MIMO $X$ network can potentially reveal more efficient schemes achieving optimality using shorter channel extensions.

\section{APPENDIX I}

\section{PRELIMINARIES}

In this section we present two lemmas that will be useful in the construction of the interference alignment schemes.

Lemma 1: Consider an $M \times M$ square matrix A such that $a_{i j}$, the element in the $i$ th row and $j$ th column of A, is of the form

$$
a_{i j}=\prod_{k=1}^{K}\left(x_{i}^{[k]}\right)^{\alpha_{i j}^{[k]}}
$$


where $x_{i}^{[k]}$ are random variables and all exponents are integers, $\alpha_{i j}^{[k]} \in \mathbb{Z}$. Suppose that

1) $x_{i}^{[k]} \mid\left\{x_{i^{\prime}}^{\left[k^{\prime}\right]}, \forall(i, k) \neq\left(i^{\prime}, k^{\prime}\right)\right\}$ has a continuous cumulative probability distribution.

2) $\forall i, j, j^{\prime} \in\{1,2, \ldots, M\}$ with $j \neq j^{\prime}$

$$
\left(\alpha_{i j}^{[1]}, \alpha_{i j}^{[2]}, \ldots, \alpha_{i j}^{[K]}\right) \neq\left(\alpha_{i j^{\prime}}^{[1]}, \alpha_{i j^{\prime}}^{[2]}, \ldots, \alpha_{i j^{\prime}}^{[K]}\right)
$$

In other words, each random variable has a continuous cdf conditioned on all the remaining variables. Also, any two terms in the same row of the matrix $\mathbf{A}$ differ in at least one exponent.

Then, the matrix $\mathbf{A}$ has a full rank of $M$ with probability 1.

Proof: We need to show that $|\mathbf{A}|$, the determinant of $\mathbf{A}$ is non-zero with probability 1 . Let $C_{i j}$ represent the co-factor corresponding to $a_{i j}$. Then,

$$
|\mathbf{A}|=C_{11} a_{11}+C_{12} a_{12} \ldots C_{1 M} a_{1 M}
$$

Note that $a_{1 j}$ is a product of powers of $x_{1}^{[k]}, k=1,2 \ldots K$. This implies that $|\mathbf{A}|=0$ only if a polynomial in $x_{1}^{[k]}, k=1,2 \ldots K$ whose co-efficients are $C_{1 j}, j=1,2 \ldots N$ is equal to zero. Therefore, $|\mathbf{A}|=0$ with non-zero probability only if atleast one of the following two conditions are satisfied.

1) The polynomial is the zero polynomial.

2) $x_{1}^{[k]}, k=1,2, \ldots, K$ are roots of the polynomial formed by setting $|\mathbf{A}|=0$.

If condition 1) is not satisfied, then the set of roots of the polynomial formed is a finite set. Notice that $C_{1 l}$ is a function of $x_{m}^{[k]}, m=2,3, \ldots, M, k=1,2, \ldots, K$. Therefore, the variables $x_{1}^{[k]}, k=1,2, \ldots, K$ have a continuous cumulative joint distribution conditioned on $C_{1 l}, l=1,2, \ldots, M$ and the probability that these variables take values from a finite set is equal to 0 . Therefore, the probability of condition 2) being satisfied is 0 . We now argue that the probability of condition 1 ) being satisfied is also equal to 0 . Now, since each $a_{1 j}$ has a unique set of exponents, condition 1) is satisfied only if all the coefficients if $a_{1 j}$ are zero, i.e., if $C_{1 j}=0, j=1,2, \ldots, M$. Therefore, $\operatorname{Pr}(|\mathbf{A}|=0)>0 \Rightarrow \operatorname{Pr}\left(C_{1 M}=0\right)>0$. Note that $C_{1 M}$ is the determinant of the matrix formed by stripping the last row and last column of $\mathbf{A}$. Now, the same argument can be iteratively used, stripping the last row and last column at each stage, until we reach a single element matrix containing $a_{M 1}$, i.e.,

$$
\operatorname{Pr}(|\mathbf{A}|=0)>0 \Rightarrow \operatorname{Pr}\left(a_{M 1}=0\right)>0
$$

$a_{M 1}$ is of the form $\prod_{k=1}^{K} x_{M}^{[k]_{M 1}^{[k]}}, k=1,2, \ldots, K$, and therefore has a continuous probability distribution. We can hence conclude that $\operatorname{Pr}\left(a_{M 1}=0\right)=0$ and therefore $|\mathbf{A}|$ is non-zero almost surely. Thus, the column vectors of $\mathbf{A}$ are linearly independent with probability 1 .

Lemma 2: Let $\mathbf{T}_{1}, \mathbf{T}_{2}, \ldots, \mathbf{T}_{N}$ be diagonal matrices of size $\mu \times \mu$ such that $T_{i j} \mid\left\{T_{i^{\prime} j^{\prime}},\left(i^{\prime}, j^{\prime}\right) \neq(i, j)\right\}$ has a continuous cumulative density function, where $T_{i j}$ represents the $j$ th diagonal entry in $T_{i}$. In other words, any diagonal entry has a continuous cumulative density function, conditioned on all the other variables forming the diagonal entries of all the matrices. Also, let $\mathbf{w}$ be a column vector which is independent of $\mathbf{T}_{1}, \mathbf{T}_{2}, \ldots, \mathbf{T}_{n}$ such that, all its entries are drawn i.i.d from a continuous distribution. Then, for any $n \in \mathbb{N}$ satisfying $\mu>(n+1)^{N}$, we can construct, with probability 1 , full rank matrices $\mathbf{V}$ and $\mathbf{V}^{\prime}$ of sizes $\mu \times n^{N}$ and $\mu \times(n+1)^{N}$ respectively, 
such that the following relations are satisfied.

$$
\begin{array}{rlr}
\operatorname{span}\left(\mathbf{T}_{1} \mathbf{V}\right) & \subset \operatorname{span}\left(\mathbf{V}^{\prime}\right) \\
\operatorname{span}\left(\mathbf{T}_{2} \mathbf{V}\right) & \subset \operatorname{span}\left(\mathbf{V}^{\prime}\right) \\
& \vdots & \\
\operatorname{span}\left(\mathbf{T}_{N} \mathbf{V}\right) & \subset \operatorname{span}\left(\mathbf{V}^{\prime}\right)
\end{array}
$$

where span $(\mathbf{P})$ represents the span of the columns of matrix $\mathbf{P}$. Furthermore, the above conditions can be satisfied with every entry in the $k$ th row of $\mathbf{V}$ ( and $\mathbf{V}^{\prime}$ ) being a multi-variate monomial function of entries in the $k$ th rows of $\mathbf{w}$ and $\mathbf{T}_{i}, i=1,2, \ldots, N$.

Proof: Let

$$
\begin{aligned}
\mathbf{V} & =\left\{\left(\prod_{i=1,2, \ldots, N} \mathbf{T}_{i}^{\alpha_{i}}\right) \mathbf{w}:\left(\alpha_{1}, \alpha_{2}, \ldots, \alpha_{N}\right) \in\{1,2, \ldots, n\}^{N}\right\} \\
\mathbf{V}^{\prime} & =\left\{\left(\prod_{i=1,2, \ldots, N} \mathbf{T}_{i}^{\alpha_{i}}\right) \mathbf{w}:\left(\alpha_{1}, \alpha_{2}, \ldots, \alpha_{N}\right) \in\{1,2, \ldots, n+1\}^{N}\right\}
\end{aligned}
$$

Note the slight abuse in notation in the above two equations - the right hand side represents the set of column vectors which may occur in any order to form the matrices on the corresponding left hand sides. To clarify the notation, let $n=1$. Then $\mathbf{V}$ contains the column vector $\mathbf{T}_{1} \mathbf{T}_{2} \ldots \mathbf{T}_{N} \mathbf{w} . \mathbf{V}^{\prime}$ contains the $2^{N}$ column vectors of the form $\mathbf{T}_{1}^{\alpha_{1}} \mathbf{T}_{2}^{\alpha_{2}} \ldots \mathbf{T}_{N}^{\alpha_{N}} \mathbf{w}$ for all $\alpha_{i} \in\{1,2\}$. It can be clearly seen that the set of columns of $\mathbf{T}_{i} \mathbf{V}$ is a subset of the set of columns of $\mathbf{V}^{\prime}$, so that

$$
\operatorname{span}\left(\mathbf{T}_{i} \mathbf{V}\right) \subset \operatorname{span}\left(\mathbf{V}^{\prime}\right), \forall i=1,2, \ldots N
$$

Further, Lemma 1 can be applied to show that $\mathbf{V}$ and $\mathbf{V}^{\prime}$ have full rank. To see this, consider the matrix

$$
\boldsymbol{\Lambda}=\left[\mathbf{V}^{\prime} \mathbf{U}\right]
$$

where $\mathbf{U}$ is a $\mu \times\left(\mu-(n+1)^{N}\right)$ matrix whose entries $u_{i j}$ are chosen i.i.d from a probability distribution whose cumulative distribution function is continuous. Then, we can apply Lemma 1 to $\Lambda$, since each term of the $l$ th row is a product of powers of $T_{m l}, u_{l k}, m=1,2 \ldots N, k=1,2 \ldots\left(\mu-(n+1)^{N}\right), l=1,2, \ldots, \mu$. Further, it can be verified that these variables and their corresponding exponents satisfy the conditions of Lemma 1. Therefore, the result of the lemma implies that $\boldsymbol{\Lambda}$ is non-singular with probability 1 . Thus, the columns of $\mathbf{V}^{\prime}$ are linearly independent almost surely. Further, since the set of columns of $\mathbf{V}$ is a subset of the columns of $\mathbf{V}^{\prime}$, the non-singularity of $\boldsymbol{\Lambda}$ implies that $\mathbf{V}$ also has linearly independent columns almost surely. This completes the proof.

\section{APPENDIX II}

\section{DEGREES OF FREEDOM OF THE $M \times N$ USER $X$ NETWORK WITH $N>2$}

The achievability scheme is similar to the achievability proof for the general $K$ user interference channel in [16]. Let $\Gamma=(M-1)(N-1)$. We will develop a coding scheme based on interference alignment which achieves a total of $\frac{N(n+1)^{\Gamma}+(M-1) N n^{\Gamma}}{N(n+1)^{\Gamma}+(M-1) n^{\Gamma}}$ degrees of freedom for any arbitrary $n$. Taking supremum over all $n$ proves that the total number of degrees of freedom is equal to $\frac{M N}{M+N-1}$ as desired. To show this, we construct a scheme that achieves a total of $(M-1) N n^{\Gamma}+N(n+1)^{\Gamma}$ degrees of freedom over a $\mu_{n}=N(n+1)^{\Gamma}+(M-1) n^{\Gamma}$ symbol extension of the original channel. (Note that, the symbol extension and the degrees of freedom achieved conform to the intuitive 
explanation provided in Section V-C) Over the extended channel, the scheme achieves $(n+1)^{\Gamma}$ degrees of freedom for each of the $N$ messages $W^{[j 1]}, j=1,2 \ldots N$ and achieves $n^{\Gamma}$ degrees of freedom for all the other $(M-1) N$ messages $W^{[j i]}, j=1,2 \ldots N, i=2,3 \ldots N$. The signal vector in the extended channel at the $j^{\text {th }}$ user's receiver can be expressed as

$$
\mathbf{Y}^{[j]}(\kappa)=\sum_{i=1}^{M} \mathbf{H}^{[j i]}(\kappa) \mathbf{X}^{[i]}(\kappa)+\mathbf{Z}^{[j]}(\kappa)
$$

where $j \in\{1,2, \ldots, N\}$, and $\mathbf{X}^{[i]}$ is a $\mu_{n} \times 1$ column vector representing the $\mu_{n}$ symbol extension of the transmitted symbol $X^{[i]}$, i.e

$$
\mathbf{X}^{[i]}(\kappa) \triangleq\left[\begin{array}{c}
X^{[i]}\left(\mu_{n} \kappa+1\right) \\
X^{[i]}\left(\mu_{n} \kappa+2\right) \\
\vdots \\
X^{[i]}\left(\mu_{n}(\kappa+1)\right)
\end{array}\right]
$$

Similarly $\mathbf{Y}^{[i]}$ and $\mathbf{Z}^{[i]}$ represent $\mu_{n}$ symbol extensions of the $Y^{[i]}$ and $Z^{[i]}$ respectively. $\mathbf{H}^{[j i]}$ is a diagonal $\mu_{n} \times \mu_{n}$ matrix representing the $\mu_{n}$ symbol extension of the channel i.e

$$
\mathbf{H}^{[j i]}(\kappa) \triangleq\left[\begin{array}{cccc}
H^{[j i]}\left(\mu_{n} \kappa+1\right) & 0 & \cdots & 0 \\
0 & H^{[j i]}\left(\mu_{n} \kappa+2\right) & \cdots & 0 \\
\vdots & \cdots & \ddots & \vdots \\
0 & 0 & \cdots & H^{[j i]}\left(\mu_{n}(\kappa+1)\right)
\end{array}\right]
$$

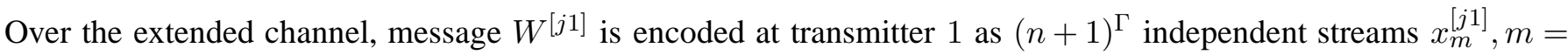
$1,2, \ldots(n+1)^{\Gamma}$ along directions $\mathbf{v}_{m}^{[j 1]}, m=1,2 \ldots(n+1)^{\Gamma}$. So the signal transmitted at transmitter 1 may be written as

$$
\mathbf{X}^{[1]}=\sum_{j=1}^{N} \sum_{m=1}^{(n+1)^{\Gamma}} x_{m}^{[j 1]} \mathbf{v}_{m}^{[j 1]}=\sum_{j=1}^{N} \mathbf{V}^{[j 1]} \mathbf{X}^{[j 1]}
$$

where the dependence on the channel use index $\kappa$ is suppressed above for the sake of compactness. Note that $\mathbf{V}^{[j 1]}$ is a $\mu_{n} \times(n+1)^{\Gamma}$ matrix whose columns are $\mathbf{v}_{m}^{[j 1]}, m=1,2, \ldots(n+1)^{\Gamma}$. Similarly $\mathbf{x}^{[j 1]}$ is a $(n+1)^{\Gamma} \times 1$ vector. In the same manner, $W^{[j i]}, i \neq 1$ is encoded into $n^{\Gamma}$ independent streams by transmitter $i$ as

$$
\mathbf{X}^{[i]}=\sum_{j=1}^{N} \sum_{m=1}^{n^{\Gamma}} x_{m}^{[j i]} \mathbf{v}_{m}^{[j i]}=\sum_{j=1}^{N} \mathbf{V}^{[j i]} \mathbf{X}^{[j i]}
$$

where $\mathbf{V}^{[j i]}$ is a $\mu_{n} \times n^{\Gamma}$ matrix The received signal at the $k^{t h}$ receiver can then be written as

$$
\mathbf{Y}^{[k]}=\sum_{i=1}^{M} \mathbf{H}^{[k i]}\left(\sum_{j=1}^{N} \mathbf{V}^{[j i]} \mathbf{X}^{[j i]}\right)+\mathbf{Z}^{[k]}
$$

We wish to design beamforming directions $\mathbf{V}^{[k i]}$ so that receiver $j$ can decode each of the desired signals by nulling the interference. We ensure this by aligning interference so that the dimension of the space spanned by the interference vectors at any receiver is equal to $(N-1)(n+1)^{\Gamma}$. Once the interference is aligned in this fashion, a receiver can decode its desired $(n+1)^{\Gamma}+(M-1) n^{\Gamma}$ streams by zero-forcing the interference in the $\mu_{n}=(N-1)(n+1)^{\Gamma}+(n+1)^{\Gamma}+(M-1) n^{\Gamma}$ dimensional space. Interference alignment is ensured by choosing 
the beamforming directions $\mathbf{V}^{[k i]}$ so that the following interference alignment equations are satisfied at receiver $j, \forall j=1,2 \ldots N$.

$$
\left.\begin{array}{ccc}
\operatorname{span}\left(\mathbf{H}^{[j i]} \mathbf{V}^{[1 i]}\right) & \subset & \operatorname{span}\left(\mathbf{H}^{[j 1]} \mathbf{V}^{[11]}\right) \\
\operatorname{span}\left(\mathbf{H}^{[j i]} \mathbf{V}^{[2 i]}\right) & \subset & \operatorname{span}\left(\mathbf{H}^{[j 1]} \mathbf{V}^{[21]}\right) \\
\operatorname{span}\left(\mathbf{H}^{[j i]} \mathbf{V}^{[(j-1) i]}\right) & \subset & \operatorname{span}\left(\mathbf{H}^{[j 1]} \mathbf{V}^{[(j-1) 1]}\right) \\
\operatorname{span}\left(\mathbf{H}^{[j i]} \mathbf{V}^{[(j+1) i]}\right) & \subset & \operatorname{span}\left(\mathbf{H}^{[j 1]} \mathbf{V}^{[(j+1) 1]}\right) \\
& \vdots & \\
\operatorname{span}\left(\mathbf{H}^{[j i]} \mathbf{V}^{[N i]}\right) & \subset & \operatorname{span}\left(\mathbf{H}^{[j 1]} \mathbf{V}^{[N 1]}\right)
\end{array}\right\} \forall i=2,3, \ldots M
$$

where $\operatorname{span}(\mathbf{P})$, represents the span of the column vectors of matrix $\mathbf{P}$. In other words, we wish to pick matrices $\mathbf{V}^{[k i]}$ so that, at receiver $j$, all the interfering spaces from transmitters $2,3, \ldots M$ align themselves with the interference from transmitter 1 . Then, at any receiver, the dimension of all the interfering streams is equal to the dimension of the interference from transmitter 1 which is equal to $(N-1)(n+1)^{\Gamma}$ as required. Note that there are $(M-1)(N-1)=\Gamma$ relations above corresponding to receiver $j$. Therefore a total of $\Gamma N$ relations of the form $\operatorname{span}(\mathbf{P}) \prec \operatorname{span}(\mathbf{Q})$ need to be satisfied. These relations can be re-ordered to be expressed alternately as

$$
\left.\begin{array}{ccc}
\operatorname{span}\left(\mathbf{H}^{[1 i]} \mathbf{V}^{[k i]}\right) & \subset & \operatorname{span}\left(\mathbf{H}^{[11]} \mathbf{V}^{[k 1]}\right) \\
\operatorname{span}\left(\mathbf{H}^{[2 i]} \mathbf{V}^{[k i]}\right) & \subset & \operatorname{span}\left(\mathbf{H}^{[21]} \mathbf{V}^{[k 1]}\right) \\
& \vdots & \\
\operatorname{span}\left(\mathbf{H}^{[(k-1) i]} \mathbf{V}^{[k i]}\right) & \subset & \operatorname{span}\left(\mathbf{H}^{[(k-1) 1]} \mathbf{V}^{[k 1]}\right) \\
\operatorname{span}\left(\mathbf{H}^{[(k+1) i]} \mathbf{V}^{[k i]}\right) & \subset & \operatorname{span}\left(\mathbf{H}^{[(k+1) 1]} \mathbf{V}^{[k 1]}\right) \\
& \vdots & \\
\operatorname{span}\left(\mathbf{H}^{[N i]} \mathbf{V}^{[k i]}\right) & \subset & \operatorname{span}\left(\mathbf{H}^{[N 1]} \mathbf{V}^{[k 1]}\right)
\end{array}\right\} \forall i=2,3, \ldots M, \forall k=1,2 \ldots N
$$

In order to satisfy the above relations, we first choose

$$
\mathbf{V}^{[k 2]}=\mathbf{V}^{[k 3]}=\ldots \mathbf{V}^{[k M]}, \forall k=1,2 \ldots N
$$

Now, the relations in (17) can be re-written as

$$
\left.\begin{array}{rrr}
\operatorname{span}\left(\mathbf{T}^{[1 i]} \mathbf{V}^{[k 2]}\right) & \subset & \operatorname{span}\left(\mathbf{V}^{[k 1]}\right) \\
\operatorname{span}\left(\mathbf{T}^{[2 i]} \mathbf{V}^{[k 2]}\right) & \subset & \operatorname{span}\left(\mathbf{V}^{[k 1]}\right) \\
\operatorname{span}\left(\mathbf{T}^{[3 i]} \mathbf{V}^{[k 2]}\right) & \subset & \operatorname{span}\left(\mathbf{V}^{[k 1]}\right) \\
& \vdots & \\
\operatorname{span}\left(\mathbf{T}^{[(k-1) i]} \mathbf{V}^{[k 2]}\right) & \subset & \operatorname{span}\left(\mathbf{V}^{[k 1]}\right) \\
\operatorname{span}\left(\mathbf{T}^{[(k+1) i]} \mathbf{V}^{[k 2]}\right) & \subset & \operatorname{span}\left(\mathbf{V}^{[k 1]}\right) \\
\operatorname{span}\left(\mathbf{T}^{[N i]} \mathbf{V}^{[k 2]}\right) & \subset & \\
\operatorname{span}\left(\mathbf{V}^{[k 1]}\right)
\end{array}\right\} \forall i=2,3 \ldots M, k=1,2 \ldots N
$$

where

$$
\mathbf{T}^{[j i]}=\left(\mathbf{H}^{[j 1]}\right)^{-1} \mathbf{H}^{[j i]}, \quad j=1,2 \ldots N, i=2,3 \ldots M
$$


We now wish to pick $\mathbf{V}^{[k 1]}$ and $\mathbf{V}^{[k 2]}$ so that the above relations are satisfied and then use $\mathbf{V}^{[k i]}=\mathbf{V}^{[k 2]}, i=$ $3,4 \ldots M$. To satisfy the above relations, we first generate $\mu_{n} \times 1$ column vectors $\mathbf{w}^{[k]}, k=1,2 \ldots N$ such that all the entries of the matrix $\left[\mathbf{w}^{[1]} \mathbf{w}^{[2]} \ldots \mathbf{w}^{[N]}\right]$ are chosen i.i.d from some continuous distribution whose support lies between a finite minimum value and a finite maximum value. Notice that for a fixed $k$, there are $\Gamma$ interference alignment relations of the form in Lemma 2. Using column vector $\mathbf{w}^{[k]}$ (which has non-zero entries with probability 1), the construction of the lemma can be used to construct vector spaces $\mathbf{V}^{[k 2]}$ and $\mathbf{V}^{[k 1]}$ satisfying the desired interference alignment relations of (18). Also, the construction ensures that $\operatorname{rank}\left(\mathbf{V}^{[k 2]}\right)=n^{\Gamma}$ and $\operatorname{rank}\left(\mathbf{V}^{[k 1]}\right)=(n+1)^{\Gamma}$ as required.

Now, we have designed $\mathbf{V}^{[j i]}, j \in\{1,2, \ldots N\}, i \in\{1,2, \ldots, M\}$ so that the desired interference alignment equations of (16) are satisfied. We now need to ensure that at each receiver, all the desired signal streams are linearly independent of each other and independent of the interference, so that they can be decoded using zero-forcing. Notice that at any receiver $k$, interference alignment ensures that all the interfering streams arrive along $\mathbf{H}^{[k 1]} \mathbf{V}^{[j 1]}, j=$ $1,2 \ldots k-1, k+1, \ldots N$ and therefore, the interference space is the space spanned by the $(N-1)(n+1)^{\Gamma}$ column vectors of $\mathbf{I}_{k}$ where

$$
\mathbf{I}_{k}=\left[\begin{array}{llllll}
\mathbf{H}^{[k 1]} \mathbf{V}^{[11]} & \mathbf{H}^{[k 1]} \mathbf{V}^{[21]} & \ldots & \mathbf{H}^{[k 1]} \mathbf{V}^{[(k-1) 1]} & \mathbf{H}^{[k 1]} \mathbf{V}^{[(k+1) 1]} & \ldots \mathbf{H}^{[k 1]} \mathbf{V}^{[N 1]}
\end{array}\right]
$$

The desired streams at receiver $k$ arrive along the $(n+1)^{\Gamma}+(M-1) n^{\Gamma}$ column vectors of $\mathbf{D}_{k}$ where

$$
\begin{aligned}
& \mathbf{D}_{k}=\left[\begin{array}{lll}
\mathbf{H}^{[k 1]} \mathbf{V}^{[k 1]} & \mathbf{H}^{[k 2]} \mathbf{V}^{[k 2]} & \ldots \mathbf{H}^{[k M]}
\end{array} \mathbf{V}^{[k M]}\right] \\
& =\left[\begin{array}{lll}
\mathbf{H}^{[k 1]} \mathbf{V}^{[k 1]} & \mathbf{H}^{[k 2]} \mathbf{V}^{[k 2]} & \ldots \mathbf{H}^{[k M]}
\end{array} \mathbf{V}^{[k 2]}\right]
\end{aligned}
$$

So, at receiver $k$, we need to ensure that the matrix

$$
\boldsymbol{\Lambda}_{k}=\left[\begin{array}{ll}
\mathbf{D}_{k} & \mathbf{I}_{k}
\end{array}\right]
$$

has a full rank of $\mu_{n}$ almost surely. Now, notice that an element in the $m$ th row of $\boldsymbol{\Lambda}_{k}$ is a product of powers of $H_{m}^{[j i]}$ and $w_{m}^{[j]}$ for $i=1,2 \ldots M, j=1,2 \ldots N$, where $H_{m}^{[j i]}$ represents the diagonal entry in the $m$ th row of $\mathbf{H}^{[j i]}$ and $w_{m}^{[j]}$ represents the entry in the $m$ th row of the column vector $\mathbf{w}^{[j]}$. We intend to use Lemma 1 to show that the matrix $\boldsymbol{\Lambda}$ has full rank with probability 1 . To do so, we need to verify that the conditions of Lemma 1 are satisfied. In particular, we need to ensure that, in a given row, the set of exponents in different columns are different. We now make the following observations

1) In the $m^{\text {th }}$ row, the product term in $\mathbf{H}^{[k i]} \mathbf{V}^{[j i]}$ contain $w_{m}^{[j]}$ with exponent 1 , but do not contain $w_{m}^{\left[j^{\prime}\right]}, j^{\prime} \neq j$

2) Notice that the equation corresponding to $\mathbf{H}^{[k i]}, i=2,3, \ldots M$ is missing in the interference alignment relations of (17) at receiver $k$. The construction of Lemma 2 ensures that monomial entries in the $m^{\text {th }}$ row of $\mathbf{V}^{[k 2]}$ do not contain $H_{m}^{[k i]}, i=2 \ldots M$.

Observation 1) implies that all the monomial entries of the $m$ th row of $\mathbf{I}_{k}$ are unique. Furthermore, it also implies that all the monomial terms in $\mathbf{I}_{k}$ are different from all the monomials in $\mathbf{D}_{k}$. Now, observation 2) implies that all the entries in $\mathbf{D}_{k}$ are unique, since the term $H_{m}^{[k i]}$ occurs only in the column vectors corresponding to $\mathbf{H}^{[k i]} \mathbf{V}^{[k 2]}$. Therefore, the conditions of Lemma 1 are met and so we can conclude the matrix $\Lambda_{k}$ has a full rank of $\mu_{n}$ almost surely.

Thus, the desired signal is linearly independent of the interference at all the receivers and therefore, using the techniques of interference alignment and zero-forcing, $\frac{N(n+1)^{\Gamma}+(M-1) N n^{\Gamma}}{N(n+1)^{\Gamma}+(M-1) n^{\Gamma}}$ degrees of freedom are achievable over 
the $M \times N$ user $X$ network for any $n \in \mathbb{N}$. Taking supremum over $n$, we conclude that the $X$ channel has $\frac{M N}{M+N-1}$ degrees of freedom.

\section{REFERENCES}

[1] G. J. Foschini and M. J. Gans, "On limits of wireless communications in a fading environment when using multiple antennas," Wireless Personal Commun. : Kluwer Academic Press, no. 6, pp. 311-335, 1998.

[2] E. Telatar, "Capacity of multi-antenna Gaussian channels," European Trans. on Telecomm. ETT, vol. 10, pp. 585-596, November 1999.

[3] D. Tse, P. Viswanath, and L. Zheng, "Diversity-multiplexing tradeoff in multiple-access channels," IEEE Trans. Inform. Theory, vol. 50, pp. 1859-1874, Sept. 2004

[4] W. Yu and J. Cioffi, "Sum capacity of Gaussian vector broadcast channels," IEEE Trans. on Information Theory, vol. 50, pp. 1875-1892, Sept. 2004.

[5] P. Viswanath and D. Tse, "On the capacity of the multiple antenna broadcast channel," in Proceedings of DIMACS workshop on Signal Processing for Wireless Transmissions, October 2002.

[6] S. Vishwanath, N. Jindal, and A. Goldsmith, "Duality, achievable rates, and sum-rate capacity of MIMO broadcast channels," IEEE Trans. Inform. Theory, pp. 2895-2909, Oct. 2003.

[7] S. Jafar and M. Fakhereddin, "Degrees of freedom for the mimo interference channel," IEEE Transactions on Information Theory, vol. 53, pp. 2637-2642, July 2007.

[8] H. Boelcskei, R. Nabar, O. Oyman, and A. Paulraj, "Capacity scaling laws in mimo relay networks," Trans. on Wireless Communications, vol. 5, pp. 1433-1444, June 2006.

[9] V. Morgenshtern, H. Boelcskei, and R. Nabar, "Distributed orthogonalization in large interference relay networks," Proceedings of IEEE Int. Symposium on Information Theory (ISIT), 2005.

[10] S. Borade, L. Zheng, and R. Gallager, "Maximizing degrees of freedom in wireless networks," in Proceedings of 40th Annual Allerton Conference on Communication, Control and Computing, pp. 561-570, October 2003.

[11] M. Maddah-Ali, A. Motahari, and A. Khandani, "Communication over MIMO X channels: Interference alignment, decomposition, and performance analysis," pp. 3457-3470, 2008.

[12] S. Jafar, "Degrees of freedom on the MIMO X channel- optimality of the MMK scheme," Tech. Report, Sep. 2006, arXiv:cs.IT/0607099v2.

[13] M. Maddah-Ali, A. Motahari, and A. Khandani, "Communication over X channel: Signalling and multiplexing gain," in Tech. Report. UW-ECE-2006-12, University of Waterloo, July 2006.

[14] M. Maddah-Ali, A. Motahari, and A. Khandani, "Communication over X channel: Signaling and performance analysis," in Tech. Report. UW-ECE-2006-27, University of Waterloo, December 2006.

[15] S. Jafar and S. Shamai, "Degrees of freedom region for the mimo X channel," IEEE Trans. on Information Theory, vol. 54, pp. 151-170, Jan. 2008.

[16] V. Cadambe and S. Jafar, "Interference alignment and the degrees of freedom of the k user interference channel," IEEE Trans. on Information Theory, vol. 54, pp. 3425-3441, Aug. 2008.

[17] A. Host-Madsen and A. Nosratinia, "The multiplexing gain of wireless networks," in Proc. of ISIT, 2005.

[18] S. Vishwanath, N. Jindal, and A. Goldsmith, "The Z channel," in Proceedings of Global Communication Conference, December 2003.

[19] G. Bresler, A. Parekh, and D. Tse, "Approximate capacity of the many-to-one interference channel," Sep. 2007. Allerton Conference.

[20] S. Sridharan, A. Jafarian, S. Vishwanath, and S. A. Jafar, "Capacity of symmetric k-user Gaussian very strong interference channels," arxiv preprint, vol. abs/0808.2314, 2008. http://arxiv.org/abs/0808.2314.

[21] S. Sridharan, A. Jafarian, S. Vishwanath, S. A. Jafar, and S. Shamai, "A layered lattice coding scheme for a class of three user Gaussian interference channels," arxiv preprint, vol. abs/0809.4316, 2008. http://arxiv.org/abs/0809.4316.

[22] "Generalized degrees of freedom of the symmetric Gaussian k user interference channel," in arXiv:cs/0804.4489 [cs.IT], 2008.

[23] H. Weingarten, S. Shamai, and G. Kramer, "On the compound MIMO broadcast channel," in Proceedings of Annual Information Theory and Applications Workshop UCSD, Jan 2007.

[24] V. Cadambe, S. Jafar, and S. Shamai, "Interference alignment on the deterministic channel and application to gaussian networks," arxiv:0711.2547.

[25] A. S. Avestimehr, S. Diggavi, and D. Tse, "A deterministic approach to wireless relay networks," Oct 2007, arXiv:cs.IT/0710.3777.

[26] N. Devroye and M. Sharif, "The multiplexing gain of MIMO X-channels with partial transmit side information," in IEEE Int. Symp. on Info. Theory (ISIT), 2007. Preprint available at the authors' website. 